J. Nonlinear Var. Anal. 4 (2020), No. 3, pp. 319-336

Available online at http://jnva.biemdas.com

https://doi.org/10.23952/jnva.4.2020.3.01

\title{
KARUSH-KUHN-TUCKER OPTIMALITY CONDITIONS AND DUALITY FOR SEMI-INFINITE PROGRAMMING PROBLEMS WITH VANISHING CONSTRAINTS
}

\author{
LE THANH TUNG \\ Department of Mathematics, College of Natural Sciences, Can Tho University, Can Tho 900000, Vietnam
}

\begin{abstract}
This paper is concerned with the semi-infinite programming problems with vanishing constraints. Both necessary and sufficient Karush-Kuhn-Tucker optimality conditions for the semi-infinite programming problems with vanishing constraints are established. We also formulate types of Wolfe and Mond-Weir dual problems and explore duality relations under convexity assumptions. Some examples are given to illustrate our results.
\end{abstract}

Keywords. Semi-infinite programming problems; Constraint qualifications; Karush-Kuhn-Tucker optimality conditions; Mond-Weir duality; Wolfe duality.

\section{INTRODUCTION}

The mathematical programming problems with vanishing constraints, presented in [1, 7], are able to be used in reformulating many problems from structural topology optimization. The papers [8, 9] considered numerous constraint qualifications and applied them to obtain corresponding Karush-Kuhn-Tucker (KKT) necessary optimality conditions. The concepts of stationary points of mathematical programming problems with vanishing constraints were studied in [3] under a topological point of view on critical point theory. Strong KKT necessary optimality conditions for multiobjective mathematical programming problems with vanishing constraints were discussed in [16]. The KKT necessary optimality conditions for mathematical programming problems with non-differentiable vanishing constraints were established in [14] via Clarke subdifferentials. Some results about duality for mathematical programming problems with vanishing constraints were obtained in $[10,15]$. On the other hand, an optimization with an infinite number of constraints is called a semi-infinite programming problem. For some recent results in this direction; see, e.g., the papers $[11,12,13,18,19,21,22,23,24]$ and references therein. In [5], KKT sufficient optimality conditions for semi-infinite programming problems with vanishing constraints were investigated. However, KKT necessary optimality conditions for semi-infinite programming problems with vanishing constraints have not considered yet in [5]. Moreover, to the best of our knowledge, there is no paper dealing with duality for semi-infinite programming problems with vanishing constraints.

Motivated by the above observations, in this paper, we establish Karush-Kuhn-Tucker optimality conditions and investigate duality problems for the semi-infinite programming problems with vanishing constraints. The outline of this paper is as follows. In Section 2, we recall basic

E-mail address: lttung@ctu.edu.vn.

Received November 29, 2019; Accepted March 19, 2020.

(C)2020 Journal of Nonlinear and Variational Analysis 
concepts and some preliminaries. The KKT necessary and sufficient optimality conditions for the semi-infinite programming problems with vanishing constraints are discussed in Section 3. Section 4 is devoted to exploring Mond-Weir and Wolfe dual problems of the semi-infinite programming problems with vanishing constraints. Some examples are provided to illustrate the outcome of the paper.

\section{PRELIMINARIES}

The following notations and definitions will be used throughout the paper. Let $\mathbb{R}^{n}$ be an Euclidean space. The notation $\langle\cdot, \cdot\rangle$ is utilized to denote the inner product. $B(x, \delta)$ indicates the open ball centered at $x$ with radius $\delta>0$. For a given $\bar{x}, \mathscr{U}(\bar{x})$ is the system of the neighborhoods of $\bar{x}$. For $A \subseteq \mathbb{R}^{n}, \operatorname{int} A, \operatorname{cl} A$, aff $A, \operatorname{span} A$ and $\operatorname{co} A$ stand for its interior, closure, affine hull, linear hull, convex hull of $A$, respectively (resp). The cone and the convex cone (containing the origin) generated by $A$ are denoted resp by $\operatorname{cone} A, \operatorname{pos} A$. Note that, for the given sets $A_{1}, A_{2}$ in $\mathbb{R}^{n}$, we can check that

$$
\operatorname{span}\left(A_{1} \cup A_{2}\right)=\operatorname{span} A_{1}+\operatorname{span} A_{2} \text { and } \operatorname{pos}\left(A_{1} \cup A_{2}\right)=\operatorname{pos} A_{1}+\operatorname{pos} A_{2} .
$$

The negative polar cone, the strictly negative polar cone and the orthogonal complement of $A$ are defined resp by

$$
\begin{gathered}
A^{-}:=\left\{x^{*} \in \mathbb{R}^{n} \mid\left\langle x^{*}, x\right\rangle \leq 0, \forall x \in A\right\}, \\
A^{s}:=\left\{x^{*} \in \mathbb{R}^{n} \mid\left\langle x^{*}, x\right\rangle<0, \forall x \in A \backslash\{0\}\right\}, \\
A^{\perp}:=\left\{x^{*} \in \mathbb{R}^{n} \mid\left\langle x^{*}, x\right\rangle=0, \forall x \in A\right\} .
\end{gathered}
$$

It is easy to check that $A^{s} \subset A^{-}$and if $A^{s} \neq \emptyset$, then $\mathrm{cl} A^{s}=A^{-}$. Moreover, the bipolar theorem (see, e.g., [2]) states that $A^{--}=\operatorname{cl}$ cone $A$. For a given nonempty subset $A$ of $\mathbb{R}^{n}$, the contingent cone [2] of $A$ at $\bar{x} \in \operatorname{cl} A$ is

$$
T(A, \bar{x}):=\left\{x \in \mathbb{R}^{n} \mid \exists \tau_{k} \downarrow 0, \exists x_{k} \rightarrow x, \forall k \in \mathbb{N}, \bar{x}+\tau_{k} x_{k} \in A\right\} .
$$

Note that if $A$ is a convex set, then $T(A, \bar{x})=\operatorname{clcone}(A-\bar{x})$. If $\left\langle x^{*}, x\right\rangle \geq 0$ for all $x^{*} \in A^{*}$, where $A^{*}$ is a subset of the dual space of $\mathbb{R}^{n}$, we write $\left\langle A^{*}, x\right\rangle \geq 0$. The notion $o\left(\tau^{k}\right)$, for $\tau>0$ and $k \in \mathbb{N}$, denotes a moving point such that $o\left(\tau^{k}\right) / \tau^{k} \rightarrow 0$ as $\tau \rightarrow 0^{+}$. The cardinality of the index set $I$ is denoted by $|I|$. For an index subset $I \subset\{1, \ldots, n\}, x_{I}=0\left(x_{I} \geq 0\right)$ stands for $x_{i}=0\left(x_{i} \geq 0\right.$, resp) for all $i \in I$.

In the line of [5], we consider the following semi-infinite programming with vanishing constraints $(\mathrm{P})$ :

$$
\begin{array}{ll}
\min & f(x) \\
\text { s.t. } & g_{t}(x) \leq 0, t \in T, \\
& h_{i}(x)=0, i=1, \ldots, q, \\
& H_{i}(x) \geq 0, i=1, \ldots, l, \\
& G_{i}(x) H_{i}(x) \leq 0, i=1, \ldots, l,
\end{array}
$$

where $f, g_{t}(t \in T), h_{i}(i=1, \ldots, q)$ and $G_{i}, H_{i}(i=1, \ldots, l)$ are continuously differentiable functions from $\mathbb{R}^{n}$ to $\mathbb{R}$. The index set $T$ is an arbitrary nonempty set, not necessary finite. Let us denote $I_{h}:=\{1, \ldots, q\}$ and $I_{l}:=\{1, \ldots, l\}$. The feasible solution set of $(\mathrm{P})$ is

$$
\Omega:=\left\{x \in \mathbb{R}^{n} \mid g_{t}(x) \leq 0(t \in T), h_{i}(x)=0\left(i \in I_{h}\right), H_{i}(x) \geq 0\left(i \in I_{l}\right), G_{i}(x) H_{i}(x) \leq 0\left(i \in I_{l}\right)\right\} .
$$


Definition 2.1. A point $\bar{x} \in \Omega$ is a local solution of (P), denoted by $\bar{x} \in \operatorname{loc} \mathscr{S}(\mathrm{P})$, if there exists a neighborhood $U \in \mathscr{U}(\bar{x})$ such that

$$
f(\bar{x}) \leq f(x), \forall x \in \Omega \cap U .
$$

If $U=\mathbb{R}^{n}$, the word "local" is omitted. In this case, the (global) solution set of (P) is denoted by $\mathscr{S}(P)$.

By $\mathbb{R}_{+}^{|T|}$, we indicate the collection of all the functions $\lambda: T \rightarrow \mathbb{R}$ taking values $\lambda_{t}$ 's positive only at finitely many points of $T$, and equal to zero at the other points. For a given $\bar{x} \in \Omega$, we designate by $I_{g}(\bar{x}):=\left\{t \in T \mid g_{t}(\bar{x})=0\right\}$ the index set of all active constraints at $\bar{x}$. The set of active constraint multipliers at $\bar{x} \in \Omega$ is

$$
\Lambda(\bar{x}):=\left\{\lambda \in \mathbb{R}_{+}^{|T|} \mid \lambda_{t} g_{t}(\bar{x})=0, \forall t \in T\right\} .
$$

Notice that $\lambda \in \Lambda(\bar{x})$ if there exists a finite index set $J \subset I_{g}(\bar{x})$ such that $\lambda_{t}>0$ for all $t \in J$ and $\lambda_{t}=0$ for all $t \in T \backslash J$. For each $\bar{x} \in \Omega$, define

$$
\begin{gathered}
I_{+}(\bar{x}):=\left\{i \in I_{l} \mid H_{i}(\bar{x})>0\right\}, I_{0}(\bar{x}):=\left\{i \in I_{l} \mid H_{i}(\bar{x})=0\right\} \\
I_{+0}(\bar{x}):=\left\{i \in I_{l} \mid H_{i}(\bar{x})>0, G_{i}(\bar{x})=0\right\} \\
I_{+-}(\bar{x}):=\left\{i \in I_{l} \mid H_{i}(\bar{x})>0, G_{i}(\bar{x})<0\right\} \\
I_{0+}(\bar{x}):=\left\{i \in I_{l} \mid H_{i}(\bar{x})=0, G_{i}(\bar{x})>0\right\} \\
I_{00}(\bar{x}):=\left\{i \in I_{l} \mid H_{i}(\bar{x})=0, G_{i}(\bar{x})=0\right\} \\
I_{0-}(\bar{x}):=\left\{i \in I_{l} \mid H_{i}(\bar{x})=0, G_{i}(\bar{x})<0\right\}
\end{gathered}
$$

Definition 2.2. Let $\bar{x} \in \Omega$.

(i) The point $\bar{x}$ is called a strong stationary point of (P) iff there exists $\left(\lambda^{g}, \lambda^{h}, \lambda^{G}, \lambda^{H}\right) \in$ $\Lambda(\bar{x}) \times \mathbb{R}^{q} \times \mathbb{R}^{l} \times \mathbb{R}^{l}$ with $\lambda_{I_{+}(\bar{x})}^{H}=0, \lambda_{I_{00}(\bar{x}) \cup I_{0-}(\bar{x})}^{H} \geq 0, \lambda_{I_{+-}(\bar{x}) \cup I_{0+}(\bar{x}) \cup I_{00}(\bar{x}) \cup I_{0-}(\bar{x})}^{G}=0$ and $\lambda_{I_{+0}(\bar{x})}^{G} \geq 0$ such that

$$
\nabla f(\bar{x})+\sum_{t \in T} \lambda_{t}^{g} \nabla g_{t}(\bar{x})+\sum_{i \in I_{h}} \lambda_{i}^{h} \nabla h_{i}(\bar{x})-\sum_{i \in I_{l}} \lambda_{i}^{H} \nabla H_{i}(\bar{x})+\sum_{i \in I_{l}} \lambda_{i}^{G} \nabla G_{i}(\bar{x})=0 .
$$

(ii) The point $\bar{x}$ is said to be a VC-stationary point of (P) iff there exists $\left(\lambda^{g}, \lambda^{h}, \lambda^{G}, \lambda^{H}\right) \in$ $\Lambda(\bar{x}) \times \mathbb{R}^{q} \times \mathbb{R}^{l} \times \mathbb{R}^{l}$ with $\lambda_{I_{+}(\bar{x})}^{H}=0, \lambda_{I_{00}(\bar{x}) \cup I_{0-}(\bar{x})}^{H} \geq 0, \lambda_{I_{+-}(\bar{x}) \cup I_{0+}(\bar{x}) \cup I_{0-}(\bar{x})}=0$ and $\lambda_{I_{+0}(\bar{x}) \cup I_{00}(\bar{x})}^{G} \geq 0$ such that

$$
\nabla f(\bar{x})+\sum_{t \in T} \lambda_{t}^{g} \nabla g_{t}(\bar{x})+\sum_{i \in I_{h}} \lambda_{i}^{h} \nabla h_{i}(\bar{x})-\sum_{i \in I_{l}} \lambda_{i}^{H} \nabla H_{i}(\bar{x})+\sum_{i \in I_{l}} \lambda_{i}^{G} \nabla G_{i}(\bar{x})=0 .
$$

It is easy to see that if $\bar{x} \in \Omega$ is a strong stationary point of $(\mathrm{P})$, then $\bar{x}$ is a VC-stationary point of $(\mathrm{P})$.

For $\bar{x} \in \Omega$ and $\left(\lambda^{g}, \lambda^{h}, \lambda^{G}, \lambda^{H}\right) \in \mathbb{R}_{+}^{|T|} \times \mathbb{R}^{q} \times \mathbb{R}^{l} \times \mathbb{R}^{l}$, define

$$
\begin{gathered}
I_{g}^{+}(\bar{x}):=\left\{t \in I_{g}(\bar{x}) \mid \lambda_{t}^{g}>0\right\}, \\
I_{h}^{+}(\bar{x}):=\left\{i \in I_{h}(\bar{x}) \mid \lambda_{i}^{h}>0\right\}, I_{h}^{-}(\bar{x}):=\left\{i \in I_{h}(\bar{x}) \mid \lambda_{i}^{h}<0\right\}, \\
\hat{I}_{+}^{+}(\bar{x}):=\left\{i \in I_{+}(\bar{x}) \mid \lambda_{i}^{H}>0\right\}, \\
\hat{I}_{0}^{+}(\bar{x}):=\left\{i \in I_{0}(\bar{x}) \mid \lambda_{i}^{H}>0\right\}, \hat{I}_{0}^{-}(\bar{x}):=\left\{i \in I_{0}(\bar{x}) \mid \lambda_{i}^{H}<0\right\},
\end{gathered}
$$




$$
\begin{aligned}
\hat{I}_{0+}^{+}(\bar{x}):=\left\{i \in I_{0+}(\bar{x}) \mid \lambda_{i}^{H}>0\right\}, \hat{I}_{0+}^{-}(\bar{x}):=\left\{i \in I_{0+}(\bar{x}) \mid \lambda_{i}^{H}<0\right\}, \\
\hat{I}_{00}^{+}(\bar{x}):=\left\{i \in I_{00}(\bar{x}) \mid \lambda_{i}^{H}>0\right\}, \hat{I}_{00}^{-}(\bar{x}):=\left\{i \in I_{00}(\bar{x}) \mid \lambda_{i}^{H}<0\right\}, \\
\hat{I}_{0-}^{+}(\bar{x}):=\left\{i \in I_{0-}(\bar{x}) \mid \lambda_{i}^{H}>0\right\}, \\
I_{+0}^{+}(\bar{x}):=\left\{i \in I_{+0}(\bar{x}) \mid \lambda_{i}^{G}>0\right\}, I_{+0}^{-}(\bar{x}):=\left\{i \in I_{+0}(\bar{x}) \mid \lambda_{i}^{G}<0\right\}, \\
I_{+-}^{+}(\bar{x}):=\left\{i \in I_{+-}(\bar{x}) \mid \lambda_{i}^{G}>0\right\}, \\
I_{0+}^{+}(\bar{x}):=\left\{i \in I_{0+}(\bar{x}) \mid \lambda_{i}^{G}>0\right\}, I_{0+}^{-}(\bar{x}):=\left\{i \in I_{0+}(\bar{x}) \mid \lambda_{i}^{G}<0\right\}, \\
I_{00}^{+}(\bar{x}):=\left\{i \in I_{00}(\bar{x}) \mid \lambda_{i}^{G}>0\right\}, I_{00}^{-}(\bar{x}):=\left\{i \in I_{00}(\bar{x}) \mid \lambda_{i}^{G}<0\right\}, \\
I_{0-}^{+}(\bar{x}):=\left\{i \in I_{0-}(\bar{x}) \mid \lambda_{i}^{G}>0\right\} .
\end{aligned}
$$

Definition 2.3. [20] Let $X \subset \mathbb{R}^{n}$ be an open convex set and $\varphi: \mathbb{R}^{n} \rightarrow \mathbb{R}$ be differentiable at $\bar{x} \in X$.

(i) $\varphi$ is convex at $\bar{x}$ if $\varphi(\lambda \bar{x}+(1-\lambda) x) \leq \lambda \varphi(\bar{x})+(1-\lambda) \varphi(x), \forall x \in X, \forall \lambda \in[0,1]$.

(ii) $\varphi$ is quasiconvex at $\bar{x}$ if $\varphi(\lambda \bar{x}+(1-\lambda) x) \leq \max \{\varphi(\bar{x}), \varphi(x)\}, \forall x \in X, \forall \lambda \in[0,1]$.

(iii) $\varphi$ is pseudoconvex at $\bar{x}$ if, for all $x \in X$,

$$
\langle\nabla \varphi(\bar{x}), x-\bar{x}\rangle \geq 0 \Rightarrow \varphi(x) \geq \varphi(\bar{x}) .
$$

(iv) $\varphi$ is convex on $X$ if $\varphi$ is convex on each point of $X$. The other concepts here introduced can be defined on a set in a similar way.

Remark 2.1. [20] Let $X \subset \mathbb{R}^{n}$ be an open convex set and $\varphi: \mathbb{R}^{n} \rightarrow \mathbb{R}$ be differentiable at $\bar{x} \in X$.

(i) If $\varphi$ is convex at $\bar{x}$, then

$$
\langle\nabla \varphi(\bar{x}), x-\bar{x}\rangle \leq \varphi(x)-\varphi(\bar{x}), \text { for all } x \in X .
$$

(ii) If $\varphi$ is quasiconvex at $\bar{x}$, then, for all $x \in X$,

$$
\varphi(x) \leq \varphi(\bar{x}) \Rightarrow\langle\nabla \varphi(\bar{x}), x-\bar{x}\rangle \leq 0 .
$$

(iii) If $\varphi$ is convex at $\bar{x}$ then $\varphi$ is pseudoconvex at $\bar{x}$. If $\varphi$ is pseudoconvex at $\bar{x}$ then $\varphi$ is quasiconvex at $\bar{x}$.

Lemma 2.1. [20] Let $\left\{C_{t} \mid t \in \Gamma\right\}$ be an arbitrary collection of nonempty convex sets in $\mathbb{R}^{n}$ and $K=\operatorname{pos}\left(\bigcup_{t \in \Gamma} C_{t}\right)$. Then, every nonzero vector of $K$ can be expressed as a non-negative linear combination of $n$ or fewer linear independent vectors, each belonging to a different $C_{t}$.

Lemma 2.2. [4] Suppose that $S, T, P$ are arbitrary (possibly infinite) index sets, $a_{s}=a(s)=$ $\left(a_{1}(s), \ldots, a_{n}(s)\right)$ maps $S$ onto $\mathbb{R}^{n}$, and so do $a_{t}$ and $a_{p}$. Suppose that the set $\operatorname{co}\left\{a_{s}, s \in S\right\}+$ $\operatorname{pos}\left\{a_{t}, t \in T\right\}+\operatorname{span}\left\{a_{p}, p \in P\right\}$ is closed. Then the following statements are equivalent:

$$
\begin{aligned}
& I:\left\{\begin{array}{l}
\left\langle a_{s}, x\right\rangle<0, s \in S, S \neq \emptyset \\
\left\langle a_{t}, x\right\rangle \leq 0, t \in T \\
\left\langle a_{p}, x\right\rangle=0, p \in P
\end{array} \quad \text { has no solution } x \in \mathbb{R}^{n} ;\right. \\
& I I: 0 \in \operatorname{co}\left\{a_{s}, s \in S\right\}+\operatorname{pos}\left\{a_{t}, t \in T\right\}+\operatorname{span}\left\{a_{p}, p \in P\right\} .
\end{aligned}
$$

Lemma 2.3. [6] If $A$ is a nonempty compact subset of $\mathbb{R}^{n}$, then,

(i) $\operatorname{co} A$ is a compact set;

(ii) if $0 \notin \operatorname{co} A$, then $\operatorname{pos} A$ is a closed cone. 


\section{KARUSH-KUHN-TUCKER OPTIMALITY CONDITIONS}

In this section, we write the index set $I_{g}$ instead of $I_{g}(\bar{x})$ for the sake of convenience. The other index sets are described similarly. Now, we establish the KKT necessary optimality condition for local solutions of $(\mathrm{P})$ under the following constraint qualifications:

(i) (ACQ):

$$
\begin{aligned}
& \left(\bigcup_{t \in I_{g}} \nabla g_{t}(\bar{x})\right)^{-} \cap\left(\bigcup_{i \in I_{h}} \nabla h_{i}(\bar{x})\right)^{\perp} \cap\left(\bigcup_{i \in I_{0+}} \nabla H_{i}(\bar{x})\right)^{\perp} \cap\left(\bigcup_{i \in I_{00} \cup I_{0-}}-\nabla H_{i}(\bar{x})\right)^{-} \cap\left(\bigcup_{i \in I_{+0}} \nabla G_{i}(\bar{x})\right)^{-} \\
& \subseteq T(\Omega, \bar{x}),
\end{aligned}
$$

(ii) (VC-ACQ):

$$
\begin{aligned}
& \left(\bigcup_{t \in I_{g}} \nabla g_{t}(\bar{x})\right)^{-} \cap\left(\bigcup_{i \in I_{h}} \nabla h_{i}(\bar{x})\right)^{\perp} \cap\left(\bigcup_{i \in I_{0+}} \nabla H_{i}(\bar{x})\right)^{\perp} \cap\left(\bigcup_{i \in I_{00} \cup I_{0-}}-\nabla H_{i}(\bar{x})\right)^{-} \cap\left(\bigcup_{i \in I_{+0} \cup I_{00}} \nabla G_{i}(\bar{x})\right)^{-} \\
& \subseteq T(\Omega, \bar{x}) .
\end{aligned}
$$

It is evident that (ACQ) implies (VC-ACQ). The following example shows that the inversion is not true in general.

Example 3.1. Let $n=2$ and $l=1$. Consider the following (P):

$$
\begin{array}{ll}
\min & f(x)=x_{1}^{2}+x_{2}^{2} \\
\mathrm{s.t.} & g_{t}(x)=-t x_{1} \leq 0, t \in T=\mathbb{N} \\
& H_{1}(x)=x_{1}+2 x_{2} \geq 0, \\
& G_{1}(x) H_{1}(x)=x_{1}\left(x_{1}+2 x_{2}\right) \leq 0
\end{array}
$$

Then,

$$
\Omega=\left\{x \in \mathbb{R}^{2} \mid x_{1} \geq 0, x_{1}+2 x_{2}=0\right\} \cup\left\{x \in \mathbb{R}^{2} \mid x_{1}=0, x_{2} \geq 0\right\} .
$$

For $\bar{x}=(0,0) \in \Omega$, one has

$$
\begin{gathered}
T(\Omega, \bar{x})=\Omega, I_{g}=\mathbb{N}, \nabla g_{t}(\bar{x})=\{(-t, 0)\}, t \in T, \\
I_{+}=I_{0+}=I_{0-}=\emptyset, I_{00}=\{1\}, \nabla G_{1}(\bar{x})=\{(1,0)\}, \nabla H_{1}(\bar{x})=\{(1,2)\}, \\
\left(\bigcup_{t \in I_{g}} \nabla g_{t}(\bar{x})\right)^{-}=\left\{x \in \mathbb{R}^{2} \mid x_{1} \geq 0\right\}, \\
\left(\bigcup_{i \in I_{00}}\left(-\nabla H_{i}(\bar{x})\right)^{-}=\left(-\nabla H_{1}(\bar{x})\right)^{-}=\left\{x \in \mathbb{R}^{2} \mid x_{1}+2 x_{2} \geq 0\right\},\right. \\
\left(\bigcup_{i \in I_{00}} \nabla G_{i}(\bar{x})\right)^{-}=\left(\nabla G_{1}(\bar{x})\right)^{-}=\left\{x \in \mathbb{R}^{2} \mid x_{1} \leq 0\right\}, \\
\left(\bigcup_{t \in I_{g}} \nabla g_{t}(\bar{x})\right)^{-} \cap\left(\bigcup_{i \in I_{00}}\left(-\nabla H_{i}(\bar{x})\right)\right)^{-}=\left\{x \in \mathbb{R}^{2} \mid x_{1} \geq 0, x_{1}+2 x_{2} \geq 0\right\},
\end{gathered}
$$

and

$$
\left(\bigcup_{t \in I_{g}} \nabla g_{t}(\bar{x})\right)^{-} \cap\left(\bigcup_{i \in I_{00}}\left(-\nabla H_{i}(\bar{x})\right)\right)^{-} \cap\left(\bigcup_{i \in I_{00}} \nabla G_{i}(\bar{x})\right)^{-}=\left\{x \in \mathbb{R}^{2} \mid x_{1}=0, x_{2} \geq 0\right\} .
$$

Hence,

$$
\left(\bigcup_{t \in I_{g}} \nabla g_{t}(\bar{x})\right)^{-} \cap\left(\bigcup_{i \in I_{00}}\left(-\nabla H_{i}(\bar{x})\right)\right)^{-} \not \subset T(\Omega, \bar{x})
$$


and

$$
\left(\bigcup_{t \in I_{g}} \nabla g_{t}(\bar{x})\right)^{-} \cap\left(\bigcup_{i \in I_{00}}\left(-\nabla H_{i}(\bar{x})\right)\right)^{-} \cap\left(\bigcup_{i \in I_{00}} \nabla G_{i}(\bar{x})\right)^{-} \subset T(\Omega, \bar{x}) .
$$

Thus, (ACQ) does not hold at $\bar{x}$ and (VC-ACQ) holds at $\bar{x}$.

Proposition 3.1. Let $\bar{x} \in \operatorname{loc} \mathscr{S}(\mathrm{P})$.

(i) If (ACQ) holds at $\bar{x}$ and the set

$$
\begin{aligned}
\Delta:= & \operatorname{pos}\left(\bigcup_{t \in I_{g}} \nabla g_{t}(\bar{x}) \cup \bigcup_{i \in I_{00} \cup I_{0-}}\left(-\nabla H_{i}(\bar{x})\right) \cup \bigcup_{i \in I_{+0}} \nabla G_{i}(\bar{x})\right) \\
& +\operatorname{span}\left(\bigcup_{i \in I_{h}} \nabla h_{i}(\bar{x}) \cup \bigcup_{i \in I_{0+}} \nabla H_{i}(\bar{x})\right)
\end{aligned}
$$

is closed, then $\bar{x}$ is a strong stationary point of $(P)$.

(ii) If (VC-ACQ) holds at $\bar{x}$ and the set

$$
\begin{aligned}
\Delta_{1}:= & \operatorname{pos}\left(\bigcup_{t \in I_{g}} \nabla g_{t}(\bar{x}) \cup \bigcup_{i \in I_{00} \cup I_{0-}}\left(-\nabla H_{i}(\bar{x})\right) \cup \bigcup_{i \in I_{+} \cup \cup I_{00}} \nabla G_{i}(\bar{x})\right) \\
& +\operatorname{span}\left(\bigcup_{i \in I_{h}} \nabla h_{i}(\bar{x}) \cup \bigcup_{i \in I_{0+}} \nabla H_{i}(\bar{x})\right)
\end{aligned}
$$

is closed, then $\bar{x}$ is a VC-stationary point of $(P)$.

Proof. Due to the similarity, we only prove (ii). Since $\bar{x} \in \operatorname{loc} \mathscr{S}(\mathrm{P})$, there exists $U \in \mathscr{U}(\bar{x})$ such that there is no $x \in \Omega \cap U$ satisfying

$$
f(x)<f(\bar{x})
$$

First, we prove that

$$
(\nabla f(\bar{x}))^{s} \cap T(\Omega, \bar{x})=\emptyset .
$$

Suppose to the contrary that there exists $d \in(\nabla f(\bar{x}))^{s} \cap T(\Omega, \bar{x})$. Then, one has

$$
\langle\nabla f(\bar{x}), d\rangle<0 .
$$

By $d \in T(\Omega, \bar{x})$, there exist $\tau_{k} \downarrow 0$ and $d_{k} \rightarrow d$ such that $\bar{x}+\tau_{k} d_{k} \in \Omega$ for all $k$. Since $f$ is continuously differentiable at $\bar{x}$, one gets

$$
f\left(\bar{x}+\tau_{k} d_{k}\right)=f(\bar{x})+\tau_{k}\left\langle\nabla f(\bar{x}), d_{k}\right\rangle+o\left(\tau_{k}\left\|d_{k}\right\|\right) .
$$

Consequently,

$$
\frac{f\left(\bar{x}+\tau_{k} d_{k}\right)-f(\bar{x})}{\tau_{k}}=\left\langle\nabla f(\bar{x}), d_{k}\right\rangle+\frac{o\left(\tau_{k}\left\|d_{k}\right\|\right)}{\tau_{k}\left\|d_{k}\right\|} \cdot\left\|d_{k}\right\| \rightarrow\langle\nabla f(\bar{x}), d\rangle<0, \text { when } k \rightarrow \infty .
$$

Thus, there exists $\bar{k}>0$ such that $\frac{f\left(\bar{x}+\tau_{k} d_{k}\right)-f(\bar{x})}{\tau_{k}}<0$, for all $k>\bar{k}$. Hence, we assure the existence of $k>\bar{k}$ large enough such that $\bar{x}+\tau_{k} d_{k} \in \Omega \cap U$ and

$$
f\left(\bar{x}+\tau_{k} d_{k}\right)<f(\bar{x}),
$$


which contradicts (3.1). Therefore, (3.2) holds. We conclude from (3.2) and (VC-ACQ) that

$$
\begin{aligned}
& (\nabla f(\bar{x}))^{s} \cap\left(\bigcup_{t \in I_{g}} \nabla g_{t}(\bar{x})\right)^{-} \cap\left(\bigcup_{i \in I_{h}} \nabla h_{i}(\bar{x})\right)^{\perp} \cap\left(\bigcup_{i \in I_{0+}} \nabla H_{i}(\bar{x})\right)^{\perp} \\
& \cap\left(\bigcup_{i \in I_{00} \cup I_{0-}}-\nabla H_{i}(\bar{x})\right)^{-} \cap\left(\bigcup_{i \in I_{+0} \cup I_{00}} \nabla G_{i}(\bar{x})\right)^{-}=\emptyset .
\end{aligned}
$$

This leads that there is no $d \in \mathbb{R}^{n}$ such that

$$
\begin{cases}\langle\nabla f(\bar{x}), d\rangle<0, & \\ \left\langle\nabla g_{t}(\bar{x}), d\right\rangle \leq 0, & \forall t \in I_{g}, \\ \left\langle\nabla h_{i}(\bar{x}), d\right\rangle=0, & \forall i \in I_{h}, \\ \left\langle\nabla H_{i}(\bar{x}), d\right\rangle=0, & \forall i \in I_{0+}, \\ \left\langle-\nabla H_{i}(\bar{x}), d\right\rangle \leq 0, & \forall i \in I_{00} \cup I_{0-}, \\ \left\langle\nabla G_{i}(\bar{x}), d\right\rangle \leq 0, & \forall i \in I_{+0} \cup I_{00} .\end{cases}
$$

On the other hand, as $\{\nabla f(\bar{x})\}$ is a compact set, $\{\nabla f(\bar{x})\}+\Delta_{1}$ is closed. According to Lemma 2.2, one has

$$
\begin{aligned}
0 \in & \nabla f(\bar{x})+\operatorname{pos}\left(\bigcup_{t \in I_{g}} \nabla g_{t}(\bar{x}) \cup \bigcup_{i \in I_{00} \cup I_{0-}}\left(-\nabla H_{i}(\bar{x})\right) \cup \bigcup_{i \in I_{+0} \cup I_{00}} \nabla G_{i}(\bar{x})\right) \\
& +\operatorname{span}\left(\bigcup_{i \in I_{h}} \nabla h_{i}(\bar{x}) \cup \bigcup_{i \in I_{0+}} \nabla H_{i}(\bar{x})\right) .
\end{aligned}
$$

Consequently,

$$
\begin{aligned}
0 \in & \nabla f(\bar{x})+\operatorname{pos} \bigcup_{t \in I_{g}} \nabla g_{t}(\bar{x})+\operatorname{span} \bigcup_{i \in I_{h}} \nabla h_{i}(\bar{x})+\operatorname{span} \bigcup_{i \in I_{0+}} \nabla H_{i}(\bar{x}) \\
& +\operatorname{pos} \bigcup_{i \in I_{00} \cup I_{0-}}\left(-\nabla H_{i}(\bar{x})\right)+\operatorname{pos} \bigcup_{i \in I_{+0} \cup I_{00}} \nabla G_{i}(\bar{x}) .
\end{aligned}
$$

From Lemma 2.1, there exists $\left(\lambda^{g}, \lambda^{h}, \lambda^{G}, \lambda^{H}\right) \in \Lambda(\bar{x}) \times \mathbb{R}^{q} \times \mathbb{R}^{l} \times \mathbb{R}^{l}$ with $\lambda_{I_{+}}^{H}=0, \lambda_{I_{00} \cup I_{0_{-}}}^{H} \geq 0$, $\lambda_{I_{+-} \cup I_{0+} \cup I_{0-}}^{G}=0$ and $\lambda_{I_{+0} \cup I_{00}}^{G} \geq 0$ such that

$$
\nabla f(\bar{x})+\sum_{t \in T} \lambda_{t}^{g} \nabla g_{t}(\bar{x})+\sum_{i \in I_{h}} \lambda_{i}^{h} \nabla h_{i}(\bar{x})-\sum_{i \in I_{l}} \lambda_{i}^{H} \nabla H_{i}(\bar{x})+\sum_{i \in I_{l}} \lambda_{i}^{G} \nabla G_{i}(\bar{x})=0 .
$$

Hence, $\bar{x}$ is a VC-stationary point of $(\mathrm{P})$.

Proposition 3.2. Let $\bar{x}$ be a strong stationary point of $(P)$. Suppose that $f$ is pseudo-convex at $\bar{x}, g_{t}\left(t \in I_{g}\right), h_{i}\left(i \in I_{h}^{+}\right),-h_{i}\left(i \in I_{h}^{-}\right), G_{i}\left(i \in I_{+0}^{+}\right), H_{i}\left(i \in \hat{I}_{0+}^{-}\right),-H_{i}\left(i \in \hat{I}_{0+}^{+} \cup \hat{I}_{00}^{+} \cup \hat{I}_{0-}^{+}\right)$are quasiconvex at $\bar{x}$. Then, the following statements hold:

(i) $\bar{x}$ is a local solution of $(P)$;

(ii) if $\hat{I}_{0+}^{-} \cup I_{+0}^{+}=\emptyset$, then $\bar{x}$ is a global solution of $(P)$.

Proof. Since $\bar{x}$ is a strong stationary point of $(\mathrm{P})$, there exists $\left(\lambda_{J}^{g}, \lambda^{h}, \lambda^{G}, \lambda^{H}\right) \in \mathbb{R}_{+}^{|J|} \times \mathbb{R}^{q} \times$ $\mathbb{R}^{l} \times \mathbb{R}^{l}$, where $J$ is a finite subset of $I_{g}$, with $\lambda_{I_{+}}^{H}=0, \lambda_{I_{00} \cup I_{0_{-}}}^{H} \geq 0, \lambda_{I_{+-} \cup I_{0+} \cup I_{00} \cup I_{0^{-}}}^{G}=0$ and 
$\lambda_{I_{+0}}^{G} \geq 0$ such that

$$
\nabla f(\bar{x})+\sum_{t \in J} \lambda_{t}^{g} \nabla g_{t}(\bar{x})+\sum_{i \in I_{h}} \lambda_{i}^{h} \nabla h_{i}(\bar{x})-\sum_{i \in I_{0+} \cup I_{00} \cup I_{0-}} \lambda_{i}^{H} \nabla H_{i}(\bar{x})+\sum_{i \in I_{+0}} \lambda_{i}^{G} \nabla G_{i}(\bar{x})=0 .
$$

For an arbitrary $x \in \Omega$, one gets that $g_{t}(x) \leq 0=g_{t}(\bar{x})$ for each $t \in I_{g}$. Thus, by the quasiconvexity at $\bar{x}$ of $g_{t}\left(t \in I_{g}\right)$, one has

$$
\left\langle\nabla g_{t}(\bar{x}), x-\bar{x}\right\rangle \leq 0, \forall t \in J
$$

which together with $\lambda_{J}^{g} \in \mathbb{R}_{+}^{|J|}$ leads to

$$
\left\langle\sum_{t \in J} \lambda_{t}^{g} \nabla g_{t}(\bar{x}), x-\bar{x}\right\rangle \leq 0 .
$$

We deduce from $x, \bar{x} \in \Omega$ that $h_{i}(x)=h_{i}(\bar{x})=0, \forall i \in I_{h}$. Hence,

$$
h_{i}(x) \leq h_{i}(\bar{x}), \forall i \in I_{h}^{+} \text {and }-h_{i}(x) \leq-h(\bar{x}), \forall i \in I_{h}^{-} .
$$

The above inequalities along with the quasiconvexity at $\bar{x}$ of $h_{i}\left(i \in I_{h}^{+}\right)$and $-h_{i}\left(i \in I_{h}^{-}\right)$implies that

$$
\left\langle\nabla h_{i}(\bar{x}), x-\bar{x}\right\rangle \leq 0, \forall i \in I_{h}^{+} \text {and }\left\langle-\nabla h_{i}(\bar{x}), x-\bar{x}\right\rangle \leq 0, \forall i \in I_{h}^{-} .
$$

It is immediate from the above inequalities and the definitions of $I_{h}^{+}, I_{h}^{-}$that

$$
\left\langle\sum_{i \in I_{h}} \lambda_{i}^{h} \nabla h_{i}(\bar{x}), x-\bar{x}\right\rangle \leq 0 .
$$

Again, we derive from $x \in \Omega$ that $-H_{i}(x) \leq 0, \forall i \in I_{l}$. Thus, $-H_{i}(x) \leq-H_{i}(\bar{x}), i \in \hat{I}_{0+}^{+} \cup \hat{I}_{00}^{+} \cup \hat{I}_{0-}^{+}$. Therefore, by the quasiconvexity of $-H_{i}, i \in \hat{I}_{0+}^{+} \cup \hat{I}_{00}^{+} \cup \hat{I}_{0-}^{+}$at $\bar{x}$, one arrives at

$$
\left\langle-\nabla H_{i}(\bar{x}), x-\bar{x}\right\rangle \leq 0, \forall i \in \hat{I}_{0+}^{+} \cup \hat{I}_{00}^{+} \cup \hat{I}_{0-}^{+} .
$$

(i) Now, for $i \in \hat{I}_{0+}^{-}$, we deduce from $G_{i}(\bar{x})>0$ and the continuity of $G_{i}$ that there exists a neighborhood $U_{1} \in \mathscr{U}(\bar{x})$ satisfying $G_{i}(x)>0$ for all $x \in U_{1}$. This leads to $H_{i}(x)=0, \forall i \in$ $\hat{I}_{0+}^{-}, \forall x \in U_{1}$. Hence, $H_{i}(x) \leq H_{i}(\bar{x}), i \in \hat{I}_{0+}^{-}$. By invoking the quasiconvexity of $H_{i}\left(i \in \hat{I}_{0+}^{-}\right)$, we have

$$
\left\langle\nabla H_{i}(\bar{x}), x-\bar{x}\right\rangle \leq 0, \forall i \in \hat{I}_{0+}^{-} .
$$

Taking into account (3.6), (3.7) and the definitions of the occurring index sets, we obtain

$$
\left\langle-\sum_{i \in I_{l}} \lambda_{i}^{H} \nabla H_{i}(\bar{x}), x-\bar{x}\right\rangle \leq 0 .
$$

Moreover, for $i \in I_{+0}^{+}$, the continuity of $H_{i}$ at $\bar{x}$ together with $H_{i}(\bar{x})>0$ justifies the existence of $U_{2} \in \mathscr{U}(\bar{x})$ such that $H_{i}(x)>0$ for all $x \in U_{2}$. Therefore, $G_{i}(x) \leq 0=G_{i}(\bar{x}), \forall i \in I_{+0}^{+}, \forall x \in U_{2}$. By employing the quasiconvexity of $G_{i}\left(i \in I_{+0}^{+}\right)$, one has $\left\langle\nabla G_{i}(\bar{x}), x-\bar{x}\right\rangle \leq 0, \forall i \in I_{+0}^{+}$. Since $\lambda_{i}^{G}>0, i \in I_{+0}^{+}$, one obtains that

$$
\left\langle\sum_{i \in I_{+0}} \lambda_{i}^{G} \nabla G_{i}(\bar{x}), x-\bar{x}\right\rangle \leq 0 .
$$


It follows from (3.3)-(3.9) that

$$
\begin{aligned}
\langle\nabla f(\bar{x}), x-\bar{x}\rangle & =-\left\langle\sum_{t \in T} \lambda_{t}^{g} \nabla g_{t}(\bar{x})+\sum_{i \in I_{h}} \lambda_{i}^{h} \nabla h_{i}(\bar{x})-\sum_{i \in I_{l}} \lambda_{i}^{H} \nabla H_{i}(\bar{x})+\sum_{i \in I_{l}} \lambda_{i}^{G} \nabla G_{i}(\bar{x}), x-\bar{x}\right\rangle \\
& \geq 0,
\end{aligned}
$$

for all $x \in \Omega \cap U$, where $U=U_{1} \cap U_{2}$. The above inequality together with the pseudo-convexity of $f$ at $\bar{x}$ implies that $f(x) \geq f(\bar{x})$ for all $x \in \Omega \cap U$.

(ii) If $\hat{I}_{0+}^{-} \cup I_{+0}^{+}=\emptyset$, then we deduce from (3.3) - (3.6) and (3.8) that

$$
\begin{aligned}
\langle\nabla f(\bar{x}), x-\bar{x}\rangle & =-\left\langle\sum_{t \in T} \lambda_{t}^{g} \nabla g_{t}(\bar{x})+\sum_{i \in I_{h}} \lambda_{i}^{h} \nabla h_{i}(\bar{x})-\sum_{i \in I_{l}} \lambda_{i}^{H} \nabla H_{i}(\bar{x})+\sum_{i \in I_{l}} \lambda_{i}^{G} \nabla G_{i}(\bar{x}), x-\bar{x}\right\rangle \\
& \geq 0,
\end{aligned}
$$

for all $x \in \Omega$. By the pseudo-convexity of $f$ at $\bar{x}$, one has $f(x) \geq f(\bar{x})$ for all $x \in \Omega$.

Proposition 3.3. Let $\bar{x}$ be a VC-stationary point of $(P)$. Suppose that $f$ is pseudo-convex at $\bar{x}$, $g_{t}\left(t \in I_{g}\right), h_{i}\left(i \in I_{h}^{+}\right),-h_{i}\left(i \in I_{h}^{-}\right), G_{i}\left(i \in I_{+0}^{+}\right), H_{i}\left(i \in \hat{I}_{0+}^{-}\right),-H_{i}\left(i \in \hat{I}_{0+}^{+} \cup \hat{I}_{00}^{+} \cup \hat{I}_{0-}^{+}\right)$are quasiconvex at $\bar{x}$. Then, the following statements hold:

(i) if $I_{00}^{+}=\emptyset, \bar{x}$ is a local solution of $(P)$;

(ii) if $\hat{I}_{0+}^{-} \cup I_{+0}^{+} \cup I_{00}^{+}=\emptyset$, then $\bar{x}$ is a global solution of $(P)$.

Proof. Since $\bar{x}$ is a VC-stationary point of (P), there exists $\left(\lambda_{J}^{g}, \lambda^{h}, \lambda^{G}, \lambda^{H}\right) \in \mathbb{R}_{+}^{|J|} \times \mathbb{R}^{q} \times \mathbb{R}^{l} \times$ $\mathbb{R}^{l}$, where $J$ is a finite subset of $I_{g}$, with $\lambda_{I_{+}}^{H}=0, \lambda_{I_{00} \cup I_{0-}}^{H} \geq 0, \lambda_{I_{+-} \cup I_{0+} \cup I_{0-}}^{G}=0$ and $\lambda_{I_{+0} \cup I_{00}}^{G} \geq 0$ such that

$$
\nabla f(\bar{x})+\sum_{t \in J} \lambda_{t}^{g} \nabla g_{t}(\bar{x})+\sum_{i \in I_{h}} \lambda_{i}^{h} \nabla h_{i}(\bar{x})-\sum_{i \in I_{0+} \cup I_{00} \cup I_{0-}} \lambda_{i}^{H} \nabla H_{i}(\bar{x})+\sum_{i \in I_{+0} \cup I_{00}} \lambda_{i}^{G} \nabla G_{i}(\bar{x})=0 .
$$

Since $I_{00}^{+}=\emptyset,(3.10)$ implies (3.3). The proof is continued just as in the proof of Proposition 3.2.

Example 3.2. Let $n=2$ and $l=1$. Consider the following (P):

$$
\begin{array}{ll}
\min & f(x)=x_{1}^{2}+x_{2}^{2}+2 x_{1}, \\
\text { s.t. } & g_{t}(x)=-t x_{1} \leq 0, t \in \mathbb{N}, \\
& H_{1}(x)=x_{1}-x_{2} \geq 0, \\
& G_{1}(x) H_{1}(x)=x_{1}\left(x_{1}-x_{2}\right) \leq 0 .
\end{array}
$$

Then, $\Omega=\left\{x \in \mathbb{R}^{2} \mid x_{1} \geq 0, x_{1}-x_{2}=0\right\} \cup\left\{x \in \mathbb{R}^{2} \mid x_{1}=0, x_{2} \leq 0\right\}$. For $\bar{x}=(0,0) \in \Omega$, one has

$$
\begin{gathered}
T(\Omega, \bar{x})=\Omega, \nabla f(\bar{x})=\{(2,0)\}, I_{g}=\mathbb{N}, \nabla g_{t}(\bar{x})=\{(-t, 0)\}, t \in T, \\
\left(\bigcup_{t \in I_{g}} \nabla g_{t}(\bar{x})\right)^{-}=\left\{x \in \mathbb{R}^{2} \mid x_{1} \geq 0\right\}, \\
I_{+}=I_{0+}=I_{0-}=\emptyset, I_{00}=\{1\}, \nabla G_{1}(\bar{x})=\{(1,0)\}, \nabla H_{1}(\bar{x})=\{(1,-1)\}, \\
\left(\bigcup_{i \in I_{00}}\left(-\nabla H_{i}(\bar{x})\right)\right)^{-}=\left\{x \in \mathbb{R}^{2} \mid x_{1}-x_{2} \geq 0\right\},\left(\bigcup_{i \in I_{00}} \nabla G_{i}(\bar{x})\right)^{-}=\left\{x \in \mathbb{R}^{2} \mid x_{1} \leq 0\right\} .
\end{gathered}
$$


Hence,

$$
\left(\bigcup_{t \in I_{g}} \nabla g_{t}(\bar{x})\right)^{-} \cap\left(\bigcup_{i \in I_{00}}\left(-\nabla H_{i}(\bar{x})\right)\right)^{-} \cap\left(\bigcup_{i \in I_{00}} \nabla G_{i}(\bar{x})\right)^{-} \subset T(\Omega, \bar{x}),
$$

i.e., (VC-ACQ) holds at $\bar{x}$. Moreover,

$$
\Delta_{1}=\operatorname{pos}\left(\bigcup_{t \in I_{g}} \nabla g_{t}(\bar{x}) \cup \bigcup_{i \in I_{00}}\left(-\nabla H_{i}(\bar{x})\right) \cup \bigcup_{i \in I_{00}} \nabla G_{i}(\bar{x})\right)=\left\{x \in \mathbb{R}^{2} \mid x_{2} \geq 0\right\}
$$

is closed. Since $f(x)-f(\bar{x}) \geq 0$ for all $x \in \Omega, \bar{x}$ is a solution of (P). Thus, all assumptions in Proposition 3.1 (ii) are satisfied. Now, let $\lambda_{1}^{H}=0, \lambda_{1}^{G}=0$ and $\lambda^{g}: T \rightarrow \mathbb{R}$ be defined by

$$
\lambda^{g}(t)= \begin{cases}1, & \text { if } t=2, \\ 0, & \text { otherwise. }\end{cases}
$$

Then,

$$
(2,0)+\sum_{t \in T} \lambda_{t}^{g}(-t, 0)-\lambda_{1}^{H}(1,-1)+\lambda_{1}^{G}(1,0)=(0,0),
$$

i.e., $\bar{x}$ is a VC-stationary point of $(\mathrm{P})$. Notice that, for the above $\left(\lambda^{g}, \lambda_{1}^{H}, \lambda_{1}^{G}\right), \bar{x}$ is also a strong stationary point of (P). Furthermore, we can check that $f, g_{t}(t \in T), H_{1},-H_{1}, G_{1}$ are convex at $\bar{x}$. Hence, all assumptions in Proposition 3.2 and Proposition 3.3 are fulfilled. Then, it follows that $\bar{x}$ is a solution of $(\mathrm{P})$. Note that if we choose $\tilde{\lambda}_{1}^{H}=0, \tilde{\lambda}_{1}^{G}=1$ and

$$
\tilde{\lambda}^{g}(t)= \begin{cases}1, & \text { if } t=3, \\ 0, & \text { otherwise }\end{cases}
$$

then

$$
(2,0)+\sum_{t \in T} \tilde{\lambda}_{t}^{g}(-t, 0)-\tilde{\lambda}_{1}^{H}(1,-1)+\tilde{\lambda}_{1}^{G}(1,0)=(0,0),
$$

i.e., $\bar{x}$ is a VC-stationary point of $(\mathrm{P})$. But, $\bar{x}$ is not a strong stationary point of $(\mathrm{P})$ in this case.

\section{The DUALity}

In this section, we consider the Wolfe [25] and Mond-Weir [17] duality schemes for (P). For $\bar{x} \in \Omega$, the index sets with respect to $\bar{x}$ are denoted identically to Section 3 .

4.1. The Wolfe type duality. For $\bar{x} \in \Omega,\left(u, \lambda^{g}, \lambda^{h}, \lambda^{G}, \lambda^{H}\right) \in \mathbb{R}^{n} \times \mathbb{R}_{+}^{|T|} \times \mathbb{R}^{q} \times \mathbb{R}^{l} \times \mathbb{R}^{l}$ with $\lambda_{I_{+}(\bar{x})}^{H} \geq 0, \lambda_{I_{0+}(\bar{x})}^{G} \leq 0$ and $\lambda_{I_{+-}(\bar{x}) \cup I_{0-}(\bar{x})}^{G} \geq 0$, we define

$$
L\left(u, \lambda^{g}, \lambda^{h}, \lambda^{G}, \lambda^{H}\right):=f(u)+\sum_{t \in T} \lambda_{t} g_{t}(u)+\sum_{i \in I_{h}} \lambda_{i}^{h} h_{i}(u)-\sum_{i \in I_{l}} \lambda_{i}^{H} H_{i}(u)+\sum_{i \in I_{l}} \lambda_{i}^{G} G_{i}(u) .
$$

In the line of [15], we consider the Wolfe type dual problem as follows:

$$
\begin{aligned}
& \left(\mathrm{D}_{W}(\bar{x})\right): \max L\left(u, \lambda^{g}, \lambda^{h}, \lambda^{G}, \lambda^{H}\right)=f(u)+\sum_{t \in T} \lambda_{t} g_{t}(u)+\sum_{i \in I_{h}} \lambda_{i}^{h} h_{i}(u) \\
& -\sum_{i \in I_{l}} \lambda_{i}^{H} H_{i}(u)+\sum_{i \in I_{l}} \lambda_{i}^{G} G_{i}(u) \\
& \text { s.t. } \nabla f(u)+\sum_{t \in T} \lambda_{t}^{g} \nabla g_{t}(u)+\sum_{i \in I_{h}} \lambda_{i}^{h} \nabla h_{i}(u)-\sum_{i \in I_{l}} \lambda_{i}^{H} \nabla H_{i}(u)+\sum_{i \in I_{l}} \lambda_{i}^{G} \nabla G_{i}(u)=0 \text {, } \\
& \lambda_{I_{+}(\bar{x})}^{H} \geq 0, \lambda_{I_{0+}(\bar{x})}^{G} \leq 0, \lambda_{I_{+-}(\bar{x}) \cup I_{0-}(\bar{x})}^{G} \geq 0, \\
& \left(u, \lambda^{g}, \lambda^{h}, \lambda^{G}, \lambda^{H}\right) \in \mathbb{R}^{n} \times \mathbb{R}_{+}^{|T|} \times \mathbb{R}^{q} \times \mathbb{R}^{l} \times \mathbb{R}^{l} .
\end{aligned}
$$


The feasible set of $\left(\mathrm{D}_{W}(\bar{x})\right)$ is defined by

$$
\begin{aligned}
\Omega_{W}(\bar{x}):=\{ & \left(u, \lambda^{g}, \lambda^{h}, \lambda^{G}, \lambda^{H}\right) \in \mathbb{R}^{n} \times \mathbb{R}_{+}^{|T|} \times \mathbb{R}^{q} \times \mathbb{R}^{l} \times \mathbb{R}^{l} \mid \lambda_{I_{+}(\bar{x})}^{H} \geq 0, \\
& \lambda_{I_{0+} G(\bar{x})}^{G} \leq 0, \lambda_{I_{+-}(\bar{x}) \cup I_{0-}(\bar{x})}^{G} \geq 0, \\
& \left.\nabla f(u)+\sum_{t \in T} \lambda_{t}^{g} \nabla g_{t}(u)+\sum_{i \in I_{h}} \lambda_{i}^{h} \nabla h_{i}(u)-\sum_{i \in I_{l}} \lambda_{i}^{H} \nabla H_{i}(u)+\sum_{i \in I_{l}} \lambda_{i}^{G} \nabla G_{i}(u)=0\right\} .
\end{aligned}
$$

We designate by

$$
\operatorname{pr}_{\mathbb{R}^{n}} \Omega_{W}(\bar{x}):=\left\{u \in \mathbb{R}^{n} \mid\left(u, \lambda^{g}, \lambda^{h}, \lambda^{G}, \lambda^{H}\right) \in \Omega_{W}(\bar{x})\right\}
$$

the projection of the set $\Omega(\bar{x})$ on $\mathbb{R}^{n}$. The other Wolfe type duality problem of $(\mathrm{P})$, which is not dependent on $\bar{x}$, is

$$
\begin{aligned}
\left(\mathrm{D}_{W}\right): \max L\left(y, \lambda^{g}, \lambda^{h}, \lambda^{G}, \lambda^{H}\right)= & f(y)+\sum_{t \in T} \lambda_{t} g_{t}(y)+\sum_{i \in I_{h}} \lambda_{i}^{h} h_{i}(y) \\
& -\sum_{i \in I_{l}} \lambda_{i}^{H} H_{i}(y)+\sum_{i \in I_{l}} \lambda_{i}^{G} G_{i}(y) \\
\text { s.t. }\left(y, \lambda^{g}, \lambda^{h}, \lambda^{G}, \lambda^{H}\right) & \in \Omega_{W}:=\bigcap_{\bar{x} \in \Omega} \Omega_{W}(\bar{x}) .
\end{aligned}
$$

Definition 4.1. Let $\bar{x} \in \Omega$.

(i) $\left(\bar{u}, \bar{\lambda}^{g}, \bar{\lambda}^{h}, \bar{\lambda}^{G}, \bar{\lambda}^{H}\right) \in \Omega_{W}(\bar{x})$ is a local solution of $\left(\mathrm{D}_{W}(\bar{x})\right)$, denoted by $\left(\bar{u}, \bar{\lambda}^{g}, \bar{\lambda}^{h}, \bar{\lambda}^{G}\right.$, $\left.\bar{\lambda}^{H}\right) \in \operatorname{loc} \mathscr{S}\left(\mathrm{D}_{W}(\bar{x})\right)$, if there exists $U \in \mathscr{N}(\bar{u})$ such that

$$
L\left(\bar{u}, \bar{\lambda}^{g}, \bar{\lambda}^{h}, \bar{\lambda}^{G}, \bar{\lambda}^{H}\right) \geq L\left(u, \lambda^{g}, \lambda^{h}, \lambda^{G}, \lambda^{H}\right), \forall\left(u, \lambda^{g}, \lambda^{h}, \lambda^{G}, \lambda^{H}\right) \in \Omega_{W}(\bar{x}) \cap U .
$$

(ii) $\left(\bar{y}, \bar{\alpha}, \bar{\lambda}^{g}, \bar{\lambda}^{h}, \bar{\lambda}^{G}, \bar{\lambda}^{H}\right) \in \Omega_{W}$ is a local solution of $\left(\mathrm{D}_{W}\right)$, denoted by $\left(\bar{y}, \bar{\lambda}^{g}, \bar{\lambda}^{h}, \bar{\lambda}^{G}, \bar{\lambda}^{H}\right) \in$ loc $\mathscr{S}\left(\mathrm{D}_{W}\right)$, if there exists $U \in \mathscr{N}(\bar{y})$ such that

$$
L\left(\bar{y}, \bar{\lambda}^{g}, \bar{\lambda}^{h}, \bar{\lambda}^{G}, \bar{\lambda}^{H}\right) \geq L\left(y, \lambda^{g}, \lambda^{h}, \lambda^{G}, \lambda^{H}\right), \forall\left(y, \lambda^{g}, \lambda^{h}, \lambda^{G}, \lambda^{H}\right) \in \Omega_{W} \cap U .
$$

If $U=\mathbb{R}^{n}$, the word "local" is omitted.

The following propositions describe weak duality relations between $(\mathrm{P})$ and the dual problems $\left(\mathrm{D}_{W}(\bar{x})\right)$ and $\left(\mathrm{D}_{W}\right)$.

Proposition 4.1. (Weak duality) Let $x \in \Omega$ and $\left(y, \lambda^{g}, \lambda^{h}, \lambda^{G}, \lambda^{H}\right) \in \Omega_{W}$. If $f$ is convex at $y$, $g_{t}\left(t \in I_{g}^{+}(x)\right), h_{i}\left(i \in I_{h}^{+}(x)\right),-h_{i}\left(i \in I_{h}^{-}(x)\right), H_{i}\left(i \in \hat{I}_{0}^{-}(x)\right),-H_{i}\left(i \in \hat{I}_{+}^{+}(x) \cup \hat{I}_{0}^{+}(x)\right), G_{i}\left(i \in I_{+0}^{+}(x) \cup\right.$ $\left.I_{+-}^{+}(x) \cup I_{00}^{+}(x) \cup I_{0-}^{+}(x)\right),-G_{i}\left(i \in I_{+0}^{-}(x) \cup I_{0+}^{-}(x) \cup I_{00}^{-}(x)\right)$ are convex at $y$, then

$$
f(x) \geq L\left(y, \lambda^{g}, \lambda^{h}, \lambda^{G}, \lambda^{H}\right) .
$$

Proof. For $x \in \Omega$ and $\left(y, \lambda^{g}, \lambda^{h}, \lambda^{G}, \lambda^{H}\right) \in \Omega_{W}=\bigcap_{\bar{x} \in \Omega} \Omega_{W}(\bar{x})$, one gets

$$
g_{t}(x) \leq 0(t \in T), h_{i}(x)=0\left(i \in I_{h}\right), H_{i}(x) \geq 0\left(i \in I_{l}\right), G_{i}(x) H_{i}(x) \leq 0\left(i \in I_{l}\right),
$$

and

$$
\nabla f(y)+\sum_{t \in T} \lambda_{t}^{g} \nabla g_{t}(y)+\sum_{i \in I_{h}} \lambda_{i}^{h} \nabla h_{i}(y)-\sum_{i \in I_{l}} \lambda_{i}^{H} \nabla H_{i}(y)+\sum_{i \in I_{l}} \lambda_{i}^{G} \nabla G_{i}(y)=0
$$

with $\lambda_{I_{+}(x)}^{H} \geq 0, \lambda_{I_{0+}(x)}^{G} \leq 0, \lambda_{I_{+-}(x) \cup I_{0-}(x)}^{G} \geq 0$. 
Therefore, we infer from (4.1), the convexity of $f, g_{t}\left(t \in I_{g}^{+}(x)\right), h_{i}\left(i \in I_{h}^{+}(x)\right),-h_{i}\left(i \in I_{h}^{-}(x)\right)$, $H_{i}\left(i \in \hat{I}_{0}^{-}(x)\right),-H_{i}\left(i \in \hat{I}_{+}^{+}(x) \cup \hat{I}_{0}^{+}(x)\right), G_{i}\left(i \in I_{+0}^{+}(x) \cup I_{+-}^{+}(x) \cup I_{00}^{+}(x) \cup I_{0-}^{+}(x)\right),-G_{i}\left(i \in I_{+0}^{-}(x) \cup\right.$ $\left.I_{0+}^{-}(x) \cup I_{00}^{-}(x)\right)$ at $y$ and the definitions of the index sets that

$$
\begin{gathered}
f(y)+\langle\nabla f(y), x-y\rangle \leq f(x), \\
g_{t}(y)+\left\langle\nabla g_{t}(y), x-y\right\rangle \leq g_{t}(x) \leq 0, \lambda_{t}^{g}>0, \forall t \in I_{g}^{+}(x), \\
h_{i}(y)+\left\langle\nabla h_{i}(y), x-y\right\rangle \leq h_{i}(x)=0, \lambda_{i}^{h}>0, \forall i \in I_{h}^{+}(x), \\
-h_{i}(y)+\left\langle-\nabla h_{i}(y), x-y\right\rangle \leq-h_{i}(x)=0, \lambda_{i}^{h}<0, \forall i \in I_{h}^{-}(x), \\
H_{i}(y)+\left\langle\nabla H_{i}(y), x-y\right\rangle \leq H_{i}(x)=0, \lambda_{i}^{H}<0, \forall i \in \hat{I}_{0}^{-}(x), \\
-H_{i}(y)+\left\langle-\nabla H_{i}(y), x-y\right\rangle \leq-H_{i}(x)<0, \lambda_{i}^{H}>0, \forall i \in \hat{I}_{+}^{+}(x), \\
-H_{i}(y)+\left\langle-\nabla H_{i}(y), x-y\right\rangle \leq-H_{i}(x)=0, \lambda_{i}^{H}>0, \forall i \in \hat{I}_{0}^{+}(x), \\
G_{i}(y)+\left\langle\nabla G_{i}(y), x-y\right\rangle \leq G_{i}(x)=0, \lambda_{i}^{G}>0, \forall i \in I_{+0}^{+}(x) \cup I_{00}^{+}(x), \\
G_{i}(y)+\left\langle\nabla G_{i}(y), x-y\right\rangle \leq G_{i}(x)<0, \lambda_{i}^{G}>0, \forall i \in I_{+-}^{+}(x) \cup I_{0-}^{+}(x), \\
-G_{i}(y)+\left\langle-\nabla G_{i}(y), x-y\right\rangle \leq-G_{i}(x)=0, \lambda_{i}^{G}<0, \forall i \in I_{+0}^{-}(x) \cup I_{00}^{-}(x),
\end{gathered}
$$

and

$$
-G_{i}(y)+\left\langle-\nabla G_{i}(y), x-y\right\rangle \leq-G_{i}(x)<0, \lambda_{i}^{G}<0, \forall i \in I_{0+}^{-}(x) .
$$

The above inequalities imply that

$$
\begin{aligned}
& f(y)+\sum_{t \in T} \lambda_{t} g_{t}(y)+\sum_{i \in I_{h}} \lambda_{i}^{h} h_{i}(y)-\sum_{i \in I_{l}} \lambda_{i}^{H} H_{i}(y)+\sum_{i \in I_{l}} \lambda_{i}^{G} G_{i}(y) \\
& +\left\langle\nabla f(y)+\sum_{t \in T} \lambda_{t}^{g} \nabla g_{t}(y)+\sum_{i \in I_{h}} \lambda_{i}^{h} \nabla h_{i}(y)-\sum_{i \in I_{l}} \lambda_{i}^{H} \nabla H_{i}(y)+\sum_{i \in I_{l}} \lambda_{i}^{G} \nabla G_{i}(y), x-y\right\rangle \leq f(x),
\end{aligned}
$$

which together with (4.2) leads to

$$
f(x) \geq L\left(y, \alpha, \lambda^{g}, \lambda^{h}, \lambda^{G}, \lambda^{H}\right) .
$$

The proof is complete.

Corollary 4.1. (Weak duality) Let $\bar{x} \in \Omega$ and $\left(u, \lambda^{g}, \lambda^{h}, \lambda^{G}, \lambda^{H}\right) \in \Omega_{W}(\bar{x})$. If $f$ is convex at $u, g_{t}\left(t \in I_{g}^{+}(\bar{x})\right), h_{i}\left(i \in I_{h}^{+}(\bar{x})\right),-h_{i}\left(i \in I_{h}^{-}(\bar{x})\right), H_{i}\left(i \in \hat{I}_{0}^{-}(\bar{x})\right),-H_{i}\left(i \in \hat{I}_{+}^{+}(\bar{x}) \cup \hat{I}_{0}^{+}(\bar{x})\right), G_{i}(i \in$ $\left.I_{+0}^{+}(\bar{x}) \cup I_{+-}^{+}(\bar{x}) \cup I_{00}^{+}(\bar{x}) \cup I_{0-}^{+}(\bar{x})\right),-G_{i}\left(i \in I_{+0}^{-}(\bar{x}) \cup I_{0+}^{-}(\bar{x}) \cup I_{00}^{-}(\bar{x})\right)$ are convex at $u$, then

$$
f(\bar{x}) \geq L\left(u, \lambda^{g}, \lambda^{h}, \lambda^{G}, \lambda^{H}\right) .
$$

Proposition 4.2. (Strong duality) Let $\bar{x} \in \Omega$ be a local solution of $(P)$. If (VC-ACQ) holds at $\bar{x}$ and the set $\Delta_{1}$ is closed, then there exists $\left(\bar{\lambda}^{g}, \bar{\lambda}^{h}, \bar{\lambda}^{G}, \bar{\lambda}^{H}\right) \in \mathbb{R}_{+}^{|T|} \times \mathbb{R}^{q} \times \mathbb{R}^{l} \times \mathbb{R}^{l}$ with $\bar{\lambda}_{I_{+}(\bar{x})}^{H}=0$, $\bar{\lambda}_{I_{00}(\bar{x}) \cup I_{0-}(\bar{x})}^{H} \geq 0, \bar{\lambda}_{I_{+-}(\bar{x}) \cup I_{0+}(\bar{x}) \cup I_{0-}(\bar{x})}=0$ and $\bar{\lambda}_{I_{+0}(\bar{x}) \cup I_{00}(\bar{x})} \geq 0$ such that $\left(\bar{x}, \bar{\lambda}^{g}, \bar{\lambda}^{h}, \bar{\lambda}^{G}, \bar{\lambda}^{H}\right) \in$ $\Omega_{W}(\bar{x})$ and $f(\bar{x})=L\left(\bar{x}, \bar{\lambda}^{g}, \bar{\lambda}^{h}, \bar{\lambda}^{G}, \bar{\lambda}^{H}\right)$. Moreover, if $f$ is convex at $\bar{x}, g_{t}\left(t \in I_{g}^{+}(\bar{x})\right), h_{i}(i \in$ $\left.I_{h}^{+}(\bar{x})\right),-h_{i}\left(i \in I_{h}^{-}(\bar{x})\right), H_{i}\left(i \in \hat{I}_{0}^{-}(\bar{x})\right),-H_{i}\left(i \in \hat{I}_{+}^{+}(\bar{x}) \cup \hat{I}_{0}^{+}(\bar{x})\right), G_{i}\left(i \in I_{+0}^{+}(\bar{x}) \cup I_{+-}^{+}(\bar{x}) \cup I_{00}^{+}(\bar{x}) \cup\right.$ $\left.I_{0-}^{+}(\bar{x})\right),-G_{i}\left(i \in I_{+0}^{-}(\bar{x}) \cup I_{0+}^{-}(\bar{x}) \cup I_{00}^{-}(\bar{x})\right)$ are convex at $\bar{x}$, then $\left(\bar{x}, \bar{\lambda}^{g}, \bar{\lambda}^{h}, \bar{\lambda}^{G}, \bar{\lambda}^{H}\right)$ is a solution of $D_{W}(\bar{x})$. 
Proof. By Proposition 3.1 (ii), there exists $\left(\bar{\lambda}^{g}, \bar{\lambda}^{h}, \bar{\lambda}^{G}, \bar{\lambda}^{H}\right) \in \Lambda(\bar{x}) \times \mathbb{R}^{q} \times \mathbb{R}^{l} \times \mathbb{R}^{l}$ with $\bar{\lambda}_{I_{+}(\bar{x})}^{H}=$ $0, \bar{\lambda}_{I_{00}(\bar{x}) \cup I_{0-}(\bar{x})}^{H} \geq 0, \bar{\lambda}_{I_{+-}(\bar{x}) \cup I_{0+}(\bar{x}) \cup I_{0-}(\bar{x})}=0$ and $\bar{\lambda}_{I_{+0}(\bar{x}) \cup I_{00}(\bar{x})}^{G} \geq 0$ such that

$$
\nabla f(\bar{x})+\sum_{t \in T} \bar{\lambda}_{t}^{g} \nabla g_{t}(\bar{x})+\sum_{i \in I_{h}} \bar{\lambda}_{i}^{h} \nabla h_{i}(\bar{x})-\sum_{i \in I_{l}} \bar{\lambda}_{i}^{H} \nabla H_{i}(\bar{x})+\sum_{i \in I_{l}} \bar{\lambda}_{i}^{G} \nabla G_{i}(\bar{x})=0 .
$$

Since $\bar{\lambda}^{g} \in \Lambda(\bar{x})$, one has $\bar{\lambda}_{t}^{g} g_{t}(\bar{x})=0$ for all $t \in T$, and thus, $\sum_{t \in T} \bar{\lambda}_{t}^{g} g_{t}(\bar{x})=0$. The fact that $\bar{x} \in \Omega$ guarantees that $\sum_{i \in I_{h}} \bar{\lambda}_{i}^{h} h_{i}(\bar{x})=0$. Moreover, we deduce from $\bar{\lambda}_{I_{+}(\bar{x})}^{H}=0$ and $H_{i}(\bar{x})=0$ for all $i \in I_{0}(\bar{x})$ that $\sum_{i \in I_{l}} \bar{\lambda}_{i}^{H} H_{i}(\bar{x})=0$. Analogously, since $\bar{\lambda}_{I_{+-}}^{G}(\bar{x}) \cup I_{0+}(\bar{x}) \cup I_{0-}(\bar{x})=0$ and $G_{i}(\bar{x})=0$ for all $i \in I_{+0}(\bar{x}) \cup I_{00}(\bar{x})$, one has $\sum_{i \in I_{l}} \bar{\lambda}_{i}^{G} G_{i}(\bar{x})=0$. Thus, $\left(\bar{x}, \bar{\lambda}^{g}, \bar{\lambda}^{h}, \bar{\lambda}^{G}, \bar{\lambda}^{H}\right) \in \Omega_{W}(\bar{x})$ and

$$
\sum_{t \in T} \lambda_{t} g_{t}(\bar{x})+\sum_{i \in I_{h}} \lambda_{i}^{h} h_{i}(\bar{x})-\sum_{i \in I_{l}} \lambda_{i}^{H} H_{i}(\bar{x})+\sum_{i \in I_{l}} \lambda_{i}^{G} G_{i}(\bar{x})=0
$$

which implies $f(\bar{x})=L\left(\bar{x}, \bar{\lambda}^{g}, \bar{\lambda}^{h}, \bar{\lambda}^{G}, \bar{\lambda}^{H}\right)$.

Now, we suppose to the contrary that $\left(\bar{x}, \bar{\lambda}^{g}, \bar{\lambda}^{h}, \bar{\lambda}^{G}, \bar{\lambda}^{H}\right)$ is not a solution of $D_{W}(\bar{x})$. By definition, there exists $\left(u, \lambda^{g}, \lambda^{h}, \lambda^{G}, \lambda^{H}\right) \in \Omega_{W}(\bar{x})$ such that

$$
L\left(\bar{x}, \bar{\lambda}^{g}, \bar{\lambda}^{h}, \bar{\lambda}^{G}, \bar{\lambda}^{H}\right)<L\left(u, \lambda^{g}, \lambda^{h}, \lambda^{G}, \lambda^{H}\right),
$$

Consequently,

$$
f(\bar{x})<L\left(u, \lambda^{g}, \lambda^{h}, \lambda^{G}, \lambda^{H}\right) .
$$

which contradicts with Corollary 4.1. So, $\left(\bar{x}, \bar{\lambda}^{g}, \bar{\lambda}^{h}, \bar{\lambda}^{G}, \bar{\lambda}^{H}\right)$ is a solution of $\left(D_{W}(\bar{x})\right)$.

Example 4.1. Let $n=2$ and $l=1$. Consider the following (P):

$$
\begin{array}{ll}
\min & f(x)=x_{1}^{2}+x_{2}^{2}+2 x_{1}, \\
\text { s.t. } & g_{t}(x)=-t x_{1} \leq 0, t \in T=\mathbb{N}, \\
& H_{1}(x)=x_{1}-x_{2} \geq 0, \\
& G_{1}(x) H_{1}(x)=x_{1}\left(x_{1}-x_{2}\right) \leq 0 .
\end{array}
$$

Then, $\Omega=\left\{x \in \mathbb{R}^{2} \mid x_{1} \geq 0, x_{1}-x_{2}=0\right\} \cup\left\{x \in \mathbb{R}^{2} \mid x_{1}=0, x_{2} \leq 0\right\}$. For any $\bar{x} \in \Omega$,

$\left(\mathrm{D}_{W}(\bar{x})\right): \max L\left(u, \lambda^{g}, \lambda^{G}, \lambda^{H}\right)=u_{1}^{2}+u_{2}^{2}+2 u_{1}-\sum_{t \in T} \lambda_{t} t u_{1}-\lambda_{1}^{H}\left(u_{1}-u_{2}\right)+\lambda_{1}^{G} u_{1}$

s.t. $\left(2 u_{1}+2, u_{2}\right)+\sum_{t \in T} \lambda_{t}^{g}(-t, 0)-\lambda_{1}^{H}(1,-1)+\lambda_{1}^{G}(1,0)=(0,0)$,

$$
\begin{aligned}
& \lambda_{1}^{H} \begin{cases}\geq 0, & \text { if } 1 \in I_{+}(\bar{x}), \quad \lambda_{1}^{G}\left\{\begin{array}{ll}
\leq 0, & \text { if } 1 \in I_{0+}(\bar{x}), \\
\in 0, & \text { if } 1 \in I_{+-}(\bar{x}) \cup I_{0-}(\bar{x}), \\
\in \mathbb{R}, & \text { if } 1 \in I_{0}(\bar{x}),
\end{array} \quad \text { if } 1 \in I_{+0}(\bar{x}) \cup I_{00}(\bar{x}),\right.\end{cases} \\
& \left(u, \lambda^{g}, \lambda_{1}^{G}, \lambda_{1}^{H}\right) \in \mathbb{R}^{2} \times \mathbb{R}_{+}^{|T|} \times \mathbb{R} \times \mathbb{R} .
\end{aligned}
$$

By taking $\bar{x}=(0,0) \in \Omega$, we invoke from Example 3.2 that all hypotheses of Proposition 4.2 are fulfilled. Now, if we select $\bar{\lambda}_{1}^{H}=0, \bar{\lambda}_{1}^{G}=0$ and

$$
\bar{\lambda}^{g}(t)= \begin{cases}1, & \text { if } t=2, \\ 0, & \text { otherwise, }\end{cases}
$$

then

$$
(2,0)+\sum_{t \in T} \bar{\lambda}_{t}^{g}(-t, 0)-\bar{\lambda}_{1}^{H}(1,-1)+\bar{\lambda}_{1}^{G}(1,0)=(0,0)
$$


i.e., $\left(\bar{x}, \bar{\lambda}^{g}, \bar{\lambda}_{1}^{G}, \bar{\lambda}_{1}^{H}\right) \in \Omega_{W}(\bar{x})$ and $f(\bar{x})=L\left(\bar{x}, \bar{\lambda}^{g}, \bar{\lambda}_{1}^{G}, \bar{\lambda}_{1}^{H}\right)$. Moreover, we can check that $f$ is convex at $\bar{x}$ and $g_{t}(t \in T), G_{1}, H_{1},-H_{1}$ are convex at $\bar{x}$. Hence, Proposition 4.2 asserts that $\left(\bar{x}, \bar{\lambda}^{g}, \bar{\lambda}_{1}^{G}, \bar{\lambda}_{1}^{H}\right)$ is a solution of $\left(\mathrm{D}_{W}(\bar{x})\right)$.

We can check directly that $\left(\bar{x}, \bar{\lambda}^{g}, \bar{\lambda}_{1}^{G}, \bar{\lambda}_{1}^{H}\right)$ is a solution of $\left(\mathrm{D}_{W}(\bar{x})\right)$ as follows. Since $\bar{x}=$ $(0,0)$, one has $I_{+}(\bar{x})=I_{0+}(\bar{x})=I_{0-}=\emptyset, I_{00}(\bar{x})=\{1\}$. Hence,

$$
\begin{gathered}
\Omega_{W}(\bar{x})=\left\{\left(u, \lambda^{g}, \lambda_{1}^{G}, \lambda_{1}^{H}\right) \in \mathbb{R}^{2} \times \mathbb{R}_{+}^{|T|} \times \mathbb{R} \times \mathbb{R} \mid \lambda_{1}^{H} \in \mathbb{R}, \lambda_{1}^{G} \in \mathbb{R},\right. \\
\left.\left(2 u_{1}+2, u_{2}\right)+\sum_{t \in T} \lambda_{t}^{g}(-t, 0)-\lambda_{1}^{H}(1,-1)+\lambda_{1}^{G}(1,0)=(0,0)\right\} .
\end{gathered}
$$

For an arbitrary $u \in \Omega_{W}(\bar{x})$, we deduce from the convexity of $f, g_{t}\left(t \in I_{g}^{+}(\bar{x})\right), G_{1}, H_{1},-H_{1}$ at $u$ and the definitions of the index sets that

$$
\begin{gathered}
f(u)+\left\langle\left(2 u_{1}+2, u_{2}\right), \bar{x}-u\right\rangle \leq f(\bar{x}), \\
g_{t}(u)+\langle(-t, 0), \bar{x}-u\rangle \leq g_{t}(\bar{x}) \leq 0, \lambda_{t}^{g}>0, \forall t \in I_{g}^{+}(\bar{x}), \\
H_{1}(u)+\langle(1,-1), \bar{x}-u\rangle \leq H_{1}(\bar{x})=0, \lambda_{1}^{H}<0, \text { if } 1 \in \hat{I}_{00}^{-}(\bar{x}), \\
-H_{1}(u)+\langle-(1,-1), \bar{x}-u\rangle \leq-H_{1}(\bar{x})=0, \lambda_{i}^{H}>0, \text { if } 1 \in \hat{I}_{00}^{+}(\bar{x}), \\
G_{1}(u)+\langle(1,0), \bar{x}-u\rangle \leq G_{1}(\bar{x})=0, \lambda_{1}^{G}>0, \text { if } 1 \in I_{00}^{+}(\bar{x}),
\end{gathered}
$$

and

$$
-G_{1}(u)+\langle-(1,0), \bar{x}-u\rangle \leq-G_{1}(\bar{x})=0, \lambda_{1}^{G}<0, \text { if } 1 \in I_{00}^{-}(\bar{x}) .
$$

The above inequalities imply that

$$
\begin{gathered}
f(u)+\sum_{t \in T} \lambda_{t} g_{t}(u)-\sum_{i \in I_{l}} \lambda_{i}^{H} H_{i}(u)+\sum_{i \in I_{l}} \lambda_{i}^{G} G_{i}(u) \\
+\left\langle\left(2 u_{1}+2, u_{2}\right)+\sum_{t \in T} \lambda_{t}^{g}(-t, 0)-\lambda_{1}^{H}(1,-1)+\lambda_{1}^{G}(1,0), \bar{x}-u\right\rangle \leq f(\bar{x}),
\end{gathered}
$$

which together with $u \in \Omega_{W}(\bar{x})$ yields that

$$
L\left(u, \lambda^{g}, \lambda_{1}^{G}, \lambda_{1}^{H}\right) \leq f(\bar{x})=L\left(\bar{x}, \bar{\lambda}^{g}, \bar{\lambda}_{1}^{G}, \bar{\lambda}_{1}^{H}\right) .
$$

4.2. The Mond-Weir type duality. For $\bar{x} \in \Omega,\left(u, \lambda^{g}, \lambda^{h}, \lambda^{G}, \lambda^{H}\right) \in \mathbb{R}^{n} \times \mathbb{R}_{+}^{|T|} \times \mathbb{R}^{q} \times \mathbb{R}^{l} \times \mathbb{R}^{l}$ with $\lambda_{I_{+}(\bar{x})}^{H} \geq 0, \lambda_{I_{0+}(\bar{x})}^{G} \leq 0$ and $\lambda_{I_{+-}^{G}(\bar{x}) \cup I_{0-}(\bar{x})} \geq 0$, we define

$$
\widetilde{L}\left(u, \lambda^{g}, \lambda^{h}, \lambda^{G}, \lambda^{H}\right):=f(u) .
$$

In the line of [15], we consider the Mond-Weir type dual problem as follows:

$\left(\mathrm{D}_{M W}(\bar{x})\right): \max \widetilde{L}\left(u, \lambda^{g}, \lambda^{h}, \lambda^{G}, \lambda^{H}\right)=f(u)$

$$
\begin{array}{ll}
\text { s.t. } & \nabla f(u)+\sum_{t \in T} \lambda_{t}^{g} \nabla g_{t}(u)+\sum_{i \in I_{h}} \lambda_{i}^{h} \nabla h_{i}(u)-\sum_{i \in I_{l}} \lambda_{i}^{H} \nabla H_{i}(u)+\sum_{i \in I_{l}} \lambda_{i}^{G} \nabla G_{i}(u)=0, \\
& \lambda_{t}^{g} g_{t}(u) \geq 0(t \in T), \lambda_{i}^{h} h_{i}(u)=0\left(i \in I_{h}\right),-\lambda_{i}^{H} H_{i}(u) \geq 0\left(i \in I_{l}\right), \lambda_{i}^{G} G_{i}(u) \geq 0\left(i \in I_{l}\right), \\
& \lambda_{I_{+}}^{H}(\bar{x}) \geq 0, \lambda_{I_{0+}(\bar{x})}^{G} \leq 0, \lambda_{I_{+-}(\bar{x}) \cup I_{0-}(\bar{x})}^{G} \geq 0, \\
& \left(u, \lambda^{g}, \lambda^{h}, \lambda^{G}, \lambda^{H}\right) \in \mathbb{R}^{n} \times \mathbb{R}_{+}^{|T|} \times \mathbb{R}^{q} \times \mathbb{R}^{l} \times \mathbb{R}^{l} .
\end{array}
$$


The feasible set of $\left(\mathrm{D}_{M W}(\bar{x})\right)$ is defined by

$$
\begin{aligned}
\Omega_{M W}(\bar{x}):=\{ & \left(u, \lambda^{g}, \lambda^{h}, \lambda^{G}, \lambda^{H}\right) \in \mathbb{R}^{n} \times \mathbb{R}_{+}^{|T|} \times \mathbb{R}^{q} \times \mathbb{R}^{l} \times \mathbb{R}^{l} \mid \lambda_{I_{+}(\bar{x})}^{H} \geq 0, \\
& \lambda_{I_{0+}(\bar{x})}^{G} \leq 0, \lambda_{I_{+-}(\bar{x}) \cup I_{0_{-}(\bar{x})} \geq 0,} \\
& \nabla f(u)+\sum_{t \in T} \lambda_{t}^{g} \nabla g_{t}(u)+\sum_{i \in I_{h}} \lambda_{i}^{h} \nabla h_{i}(u)-\sum_{i \in I_{l}} \lambda_{i}^{H} \nabla H_{i}(u)+\sum_{i \in I_{l}} \lambda_{i}^{G} \nabla G_{i}(u)=0, \\
& \lambda_{t}^{g} g_{t}(u) \geq 0(t \in T), \lambda_{i}^{h} h_{i}(u)=0\left(i \in I_{h}\right), \\
& \left.-\lambda_{i}^{H} H_{i}(u) \geq 0\left(i \in I_{l}\right), \lambda_{i}^{G} G_{i}(u) \geq 0\left(i \in I_{l}\right)\right\} .
\end{aligned}
$$

We designate by

$$
\operatorname{pr}_{\mathbb{R}^{n}} \Omega_{M W}(\bar{x}):=\left\{u \in \mathbb{R}^{n} \mid\left(u, \lambda^{g}, \lambda^{h}, \lambda^{G}, \lambda^{H}\right) \in \Omega_{M W}(\bar{x})\right\}
$$

the projection of the set $\Omega_{M W}(\bar{x})$ on $\mathbb{R}^{n}$. The other Mond-Weir type duality problem of (P), which is not dependent on $\bar{x}$, is

$$
\begin{aligned}
& \left(\mathrm{D}_{M W}\right): \max \widetilde{L}\left(y, \lambda^{g}, \lambda^{h}, \lambda^{G}, \lambda^{H}\right)=f(y) \\
& \text { s.t. }\left(y, \lambda^{g}, \lambda^{h}, \lambda^{G}, \lambda^{H}\right) \in \Omega_{M W}:=\bigcap_{\bar{x} \in \Omega} \Omega_{M W}(\bar{x}) .
\end{aligned}
$$

Definition 4.2. Let $\bar{x} \in \Omega$.

(i) $\left(\bar{u}, \bar{\lambda}^{g}, \bar{\lambda}^{h}, \bar{\lambda}^{G}, \bar{\lambda}^{H}\right) \in \Omega_{M W}(\bar{x})$ is a local solution of $\left(\mathrm{D}_{M W}(\bar{x})\right)$, denoted by $\left(\bar{u}, \bar{\lambda}^{g}, \bar{\lambda}^{h}, \bar{\lambda}^{G}\right.$, $\left.\bar{\lambda}^{H}\right) \in \operatorname{loc} \mathscr{S}\left(\mathrm{D}_{M W}(\bar{x})\right)$, if there exists $U \in \mathscr{N}(\bar{u})$ such that

$\widetilde{L}\left(\bar{u}, \bar{\lambda}^{g}, \bar{\lambda}^{h}, \bar{\lambda}^{G}, \bar{\lambda}^{H}\right) \geq \widetilde{L}\left(u, \lambda^{g}, \lambda^{h}, \lambda^{G}, \lambda^{H}\right), \forall\left(u, \lambda^{g}, \lambda^{h}, \lambda^{G}, \lambda^{H}\right) \in \Omega_{M W}(\bar{x}) \cap U$.

(ii) $\left(\bar{y}, \bar{\lambda}^{g}, \bar{\lambda}^{h}, \bar{\lambda}^{G}, \bar{\lambda}^{H}\right) \in \Omega_{M W}$ is a local solution of $\left(\mathrm{D}_{M W}\right)$, denoted by $\left(\bar{y}, \bar{\lambda}^{g}, \bar{\lambda}^{h}, \bar{\lambda}^{G}\right.$, $\left.\bar{\lambda}^{H}\right) \in \operatorname{loc} \mathscr{S}\left(\mathrm{D}_{M W}\right)$, if there exists $U \in \mathscr{N}(\bar{y})$ such that

$$
\widetilde{L}\left(\bar{y}, \bar{\lambda}^{g}, \bar{\lambda}^{h}, \bar{\lambda}^{G}, \bar{\lambda}^{H}\right) \geq \widetilde{L}\left(y, \lambda^{g}, \lambda^{h}, \lambda^{G}, \lambda^{H}\right), \forall\left(y, \lambda^{g}, \lambda^{h}, \lambda^{G}, \lambda^{H}\right) \in \Omega_{M W} \cap U .
$$

If $U=\mathbb{R}^{n}$, then the word "local" is dropped.

Proposition 4.3. (Weak duality) Let $x \in \Omega$ and $\left(y, \lambda^{g}, \lambda^{h}, \lambda^{G}, \lambda^{H}\right) \in \Omega_{M W}$. If $f$ is pseudoconvex at $y, g_{t}\left(t \in I_{g}^{+}(x)\right), h_{i}\left(i \in I_{h}^{+}(x)\right),-h_{i}\left(i \in I_{h}^{-}(x)\right), H_{i}\left(i \in \hat{I}_{0}^{-}(x)\right),-H_{i}\left(i \in \hat{I}_{+}^{+}(x) \cup \hat{I}_{0}^{+}(x)\right), G_{i}(i \in$ $\left.I_{+0}^{+}(x) \cup I_{+-}^{+}(x) \cup I_{00}^{+}(x)\right),-G_{i}\left(i \in I_{+0}^{-}(x) \cup I_{0+}^{-}(x) \cup I_{00}^{-}(x)\right)$ are quasiconvex at $y$, then

$$
f(x) \geq \widetilde{L}\left(y, \lambda^{g}, \lambda^{h}, \lambda^{G}, \lambda^{H}\right) .
$$

Proof. For $x \in \Omega$ and $\left(y, \lambda^{g}, \lambda^{h}, \lambda^{G}, \lambda^{H}\right) \in \Omega_{M W}=\bigcap_{\bar{x} \in \Omega} \Omega_{M W}(\bar{x})$, one gets

$$
\begin{aligned}
& g_{t}(x) \leq 0(t \in T), h_{i}(x)=0\left(i \in I_{h}\right), H_{i}(x) \geq 0\left(i \in I_{l}\right), G_{i}(x) H_{i}(x) \leq 0\left(i \in I_{l}\right), \\
& \nabla f(y)+\sum_{t \in T} \lambda_{t}^{g} \nabla g_{t}(y)+\sum_{i \in I_{h}} \lambda_{i}^{h} \nabla h_{i}(y)-\sum_{i \in I_{l}} \lambda_{i}^{H} \nabla H_{i}(y)+\sum_{i \in I_{l}} \lambda_{i}^{G} \nabla G_{i}(y)=0,
\end{aligned}
$$

and

$$
\lambda_{t}^{g} g_{t}(y) \geq 0(t \in T), \lambda_{i}^{h} h_{i}(y)=0\left(i \in I_{h}\right),-\lambda_{i}^{H} H_{i}(y) \geq 0\left(i \in I_{l}\right), \lambda_{i}^{G} G_{i}(y) \geq 0\left(i \in I_{l}\right),
$$

with $\lambda_{I_{+}(x)}^{H} \geq 0, \lambda_{I_{0+}(x)}^{G} \leq 0$, and $\lambda_{I_{+-}(x) \cup I_{0-}(x)}^{G} \geq 0$. It follows from the above inequalities that

$$
g_{t}(x) \leq 0 \leq g_{t}(y), \forall t \in I_{g}^{+}(x),
$$




$$
\begin{gathered}
h_{i}(x)=h_{i}(y)=0, \forall i \in I_{h}^{+}(x) \cup I_{h}^{-}(x), \\
H_{i}(x)=0 \leq H_{i}(y), \forall i \in \hat{I}_{0}^{-}(x), \\
-H_{i}(x) \leq 0 \leq-H_{i}(y), \forall i \in \hat{I}_{+}^{+}(x) \cup \hat{I}_{0}^{+}(x), \\
G_{i}(x) \leq 0 \leq G_{i}(y), \forall i \in I_{+0}^{+}(x) \cup I_{+-}^{+}(x) \cup I_{00}^{+}(x) \cup I_{0-}^{+}(x),
\end{gathered}
$$

and

$$
-G_{i}(x) \leq 0 \leq-G_{i}(y), \forall i \in I_{+0}^{-}(x) \cup I_{0+}^{-}(x) \cup I_{00}^{-}(x) .
$$

Therefore, we deduce from the quasiconvexity of $g_{t}\left(t \in I_{g}^{+}(x)\right), h_{i}\left(i \in I_{h}^{+}(x)\right),-h_{i}\left(i \in I_{h}^{-}(x)\right)$, $H_{i}\left(i \in \hat{I}_{0}^{-}(x)\right),-H_{i}\left(i \in \hat{I}_{+}^{+}(x) \cup \hat{I}_{0}^{+}(x)\right), G_{i}\left(i \in I_{+0}^{+}(x) \cup I_{+-}^{+}(x) \cup I_{00}^{+}(x)\right),-G_{i}\left(i \in I_{+0}^{-}(x) \cup I_{0+}^{-}(x) \cup\right.$ $\left.I_{00}^{-}(x)\right)$ at $y$ and the definitions of the index sets that

$$
\begin{gathered}
\left\langle\nabla g_{i}(y), x-y\right\rangle \leq 0, \lambda_{i}^{g}>0, \forall i \in I_{g}^{+}(x), \\
\left\langle\nabla h_{i}(y), x-y\right\rangle \leq 0, \lambda_{i}^{h}>0, \forall i \in I_{h}^{+}(x), \\
\left\langle-\nabla h_{i}(y), x-y\right\rangle \leq 0, \lambda_{i}^{h}<0, \forall i \in I_{h}^{-}(x), \\
\left\langle\nabla H_{i}(y), x-y\right\rangle \leq 0, \lambda_{i}^{H}<0, \forall i \in \hat{I}_{0}^{-}(x), \\
\left\langle-\nabla H_{i}(y), x-y\right\rangle \leq 0, \lambda_{i}^{H}>0, \forall i \in \hat{I}_{+}^{+}(x) \cup \hat{I}_{0}^{+}(x), \\
\left\langle\nabla G_{i}(y), x-y\right\rangle \leq 0, \lambda_{i}^{G}>0, \forall i \in I_{+0}^{+}(x) \cup I_{+-}^{+}(x) \cup I_{00}^{+}(x) \cup I_{0-}^{+}(x),
\end{gathered}
$$

and

$$
\left\langle-\nabla G_{i}(y), x-y\right\rangle \leq 0, \lambda_{i}^{G}<0, \forall i \in I_{+0}^{-}(x) \cup I_{0+}^{-}(x) \cup I_{00}^{-}(x) .
$$

We derive from the above inequalities and (4.4) that

$$
\langle\nabla f(y), x-y\rangle=-\left\langle\sum_{t \in T} \lambda_{t}^{g} \nabla g_{t}(y)+\sum_{i \in I_{h}} \lambda_{i}^{h} \nabla h_{i}(y)-\sum_{i \in I_{l}} \lambda_{i}^{H} \nabla H_{i}(y)+\sum_{i \in I_{l}} \lambda_{i}^{G} \nabla G_{i}(y), x-y\right\rangle \geq 0,
$$

which along with the pseudoconvexity of $f$ at $y$ implies that

$$
f(x) \geq f(y)=\widetilde{L}\left(y, \lambda^{g}, \lambda^{h}, \lambda^{G}, \lambda^{H}\right) .
$$

The conclusion is obtained.

Corollary 4.2. (Weak duality) Let $\bar{x} \in \Omega$ and $\left(u, \lambda^{g}, \lambda^{h}, \lambda^{G}, \lambda^{H}\right) \in \Omega_{M W}(\bar{x})$. If $f$ is pseudoconvexat $u, g_{t}\left(t \in I_{g}^{+}(\bar{x})\right), h_{i}\left(i \in I_{h}^{+}(\bar{x})\right),-h_{i}\left(i \in I_{h}^{-}(\bar{x})\right), H_{i}\left(i \in \hat{I}_{0}^{-}(\bar{x})\right),-H_{i}\left(i \in \hat{I}_{+}^{+}(\bar{x}) \cup \hat{I}_{0}^{+}(\bar{x})\right), G_{i}(i \in$ $\left.I_{+0}^{+}(\bar{x}) \cup I_{+-}^{+}(\bar{x}) \cup I_{00}^{+}(\bar{x})\right),-G_{i}\left(i \in I_{+0}^{-}(\bar{x}) \cup I_{0+}^{-}(\bar{x}) \cup I_{00}^{-}(\bar{x})\right)$ are quasiconvex at $u$, then

$$
f(\bar{x}) \geq \widetilde{L}\left(u, \lambda^{g}, \lambda^{h}, \lambda^{G}, \lambda^{H}\right) .
$$

Proposition 4.4. (strong duality) Let $\bar{x} \in \Omega$ be a local solution of $(P)$. If (VC-ACQ) holds at $\bar{x}$ and the set $\Delta_{1}$ is closed, then there exists $\left(\bar{\lambda}^{g}, \bar{\lambda}^{h}, \bar{\lambda}^{G}, \bar{\lambda}^{H}\right) \in \mathbb{R}_{+}^{|T|} \times \mathbb{R}^{q} \times \mathbb{R}^{l} \times \mathbb{R}^{l}$ with $\bar{\lambda}_{I_{+}(\bar{x})}^{H}=0$, $\bar{\lambda}_{I_{00}(\bar{x}) \cup I_{0-}(\bar{x})} \geq 0, \bar{\lambda}_{I_{+-}(\bar{x}) \cup I_{0+}(\bar{x}) \cup I_{0-}(\bar{x})}=0$ and $\bar{\lambda}_{I_{+0}(\bar{x}) \cup I_{00}(\bar{x})} \geq 0$ such that $\left(\bar{x}, \bar{\lambda}^{g}, \bar{\lambda}^{h}, \bar{\lambda}^{G}, \bar{\lambda}^{H}\right) \in$ $\Omega_{M W}(\bar{x})$. Moreover, if $f$ is pseudoconvex at $\bar{x}, g_{t}\left(t \in I_{g}^{+}(\bar{x})\right), h_{i}\left(i \in I_{h}^{+}(\bar{x})\right),-h_{i}\left(i \in I_{h}^{-}(\bar{x})\right), H_{i}(i \in$ $\left.\hat{I}_{0}^{-}(\bar{x})\right),-H_{i}\left(i \in \hat{I}_{+}^{+}(\bar{x}) \cup \hat{I}_{0}^{+}(\bar{x})\right), G_{i}\left(i \in I_{+0}^{+}(\bar{x}) \cup I_{+-}^{+}(\bar{x}) \cup I_{00}^{+}(\bar{x})\right),-G_{i}\left(i \in I_{+0}^{-}(\bar{x}) \cup I_{0+}^{-}(\bar{x}) \cup I_{00}^{-}(\bar{x})\right)$ are quasiconvex at $\bar{x}$, then $\left(\bar{x}, \bar{\lambda}^{g}, \bar{\lambda}^{h}, \bar{\lambda}^{G}, \bar{\lambda}^{H}\right)$ is a solution of $D_{M W}(\bar{x})$. 
Proof. By Proposition 3.1 (ii), there exists $\left(\bar{\lambda}^{g}, \bar{\lambda}^{h}, \bar{\lambda}^{G}, \bar{\lambda}^{H}\right) \in \Lambda(\bar{x}) \times \mathbb{R}^{q} \times \mathbb{R}^{l} \times \mathbb{R}^{l}$ with $\bar{\lambda}_{I_{+}(\bar{x})}^{H}=$ $0, \bar{\lambda}_{I_{00}(\bar{x}) \cup I_{0-}(\bar{x})}^{H} \geq 0, \bar{\lambda}_{I_{+-}(\bar{x}) \cup I_{0+}(\bar{x}) \cup I_{0-}(\bar{x})}=0$ and $\bar{\lambda}_{I_{+0}(\bar{x}) \cup I_{00}(\bar{x})}^{G} \geq 0$ such that

$$
\nabla f(\bar{x})+\sum_{t \in T} \bar{\lambda}_{t}^{g} \nabla g_{t}(\bar{x})+\sum_{i \in I_{h}} \bar{\lambda}_{i}^{h} \nabla h_{i}(\bar{x})-\sum_{i \in I_{l}} \bar{\lambda}_{i}^{H} \nabla H_{i}(\bar{x})+\sum_{i \in I_{l}} \bar{\lambda}_{i}^{G} \nabla G_{i}(\bar{x})=0 .
$$

Since $\bar{\lambda}^{g} \in \Lambda(\bar{x})$, one has $\bar{\lambda}_{t}^{g} g_{t}(\bar{x})=0$ for all $t \in T$. It follows from $\bar{x} \in \Omega$ that $\lambda_{i}^{h} h_{i}(\bar{x})=0, \forall i \in$ $I_{h}$. Moreover, we deduce from $\bar{\lambda}_{I_{+}(\bar{x})}^{H}=0$ and $H_{i}(\bar{x})=0$ for all $i \in I_{0}(\bar{x})$ that $-\bar{\lambda}_{i}^{H} H_{i}(\bar{x})=0$ for all $i \in I_{l}$. Similarly, since $\bar{\lambda}_{I_{I_{-}}(\bar{x}) \cup I_{0+}(\bar{x}) \cup I_{0-}(\bar{x})}=0$ and $G_{i}(\bar{x})=0$ for all $i \in I_{+0}(\bar{x}) \cup I_{00}(\bar{x})$, one has $\bar{\lambda}_{i}^{G} G_{i}(\bar{x})=0$ for all $i \in I_{l}$. Thus, $\left(\bar{x}, \bar{\lambda}^{g}, \bar{\lambda}^{h}, \bar{\lambda}^{G}, \bar{\lambda}^{H}\right) \in \Omega_{M W}(\bar{x})$ and $f(\bar{x})=\widetilde{L}\left(\bar{x}, \bar{\lambda}^{g}, \bar{\lambda}^{h}, \bar{\lambda}^{G}, \bar{\lambda}^{H}\right)$. Arguing by contradiction, we suppose that $\left(\bar{x}, \bar{\lambda}^{g}, \bar{\lambda}^{h}, \bar{\lambda}^{G}, \bar{\lambda}^{H}\right)$ is not a solution of $D_{M W}(\bar{x})$. By denotation, there exists $\left(u, \lambda^{g}, \lambda^{h}, \lambda^{G}, \lambda^{H}\right) \in \Omega_{M W}(\bar{x})$ such that

$$
f(\bar{x})=\widetilde{L}\left(\bar{x}, \bar{\lambda}^{g}, \bar{\lambda}^{h}, \bar{\lambda}^{G}, \bar{\lambda}^{H}\right)<\widetilde{L}\left(u, \lambda^{g}, \lambda^{h}, \lambda^{G}, \lambda^{H}\right),
$$

which contradicts Corollary 4.2. So, $\left(\bar{x}, \bar{\lambda}^{g}, \bar{\lambda}^{h}, \bar{\lambda}^{G}, \bar{\lambda}^{H}\right)$ is a solution of $D_{M W}(\bar{x})$. This completes the proof.

\section{REFERENCES}

[1] W. Achtziger, C. Kanzow, Mathematical programs with vanishing constraints: optimality conditions and constraint qualifications, Math. Program. 114 (2007), 69-99.

[2] J.P. Aubin, H. Frankowska, Set-Valued Analysis, Birkhäuser, Boston, 1990.

[3] D. Dorsch, V. Shikhman, O. Stein, Mathematical programs with vanishing constraints: critical point theory, J. Glob. Optim. 52 (2012), 591-605.

[4] M.A. Goberna, M.A. Lopéz, Linear Semi-Infinite Optimization, Wiley, Chichester, 1998.

[5] S.M. Guu, Y. Singh, S.K. Mishra, On strong KKT type sufficient optimality conditions for multiobjective semi-infinite programming problems with vanishing constraints, J. Ineq. Appl. 2017 (2017), 1-9.

[6] J.B. Hiriart-Urruty, C. Lemaréchal, Convex Analysis and Minimization Algorithms I, Springer, Berlin, 1993.

[7] T. Hoheisel, C. Kanzow, First- and second-order optimality conditions for mathematical programs with vanishing constraints, Appl. Math. 52 (2007), 495-514.

[8] T. Hoheisel, C. Kanzow, Stationarity conditions for mathematical programs with vanishing constraints using weak constraint qualifications, J. Math. Anal. Appl. 337 (2008), 292-310.

[9] T. Hoheisel, C. Kanzow, On the Abadie and Guignard constraint qualifications for mathematical programs with vanishing constraints, Optimization 58 (2009), 431-448.

[10] Q. Hu, J. Wang, Y. Chen, New dualities for mathematical programs with vanishing constraints, Annal. Oper. Res. 287 (2020), 233-255.

[11] A. Kabgani, M. Soleimani-damaneh, Characterization of (weakly/properly/robust) efficient solutions in nonsmooth semi-infinite multiobjective optimization using convexificators, Optimization 67 (2017), 217-235.

[12] N. Kanzi, S. Nobakhtian, Optimality conditions for nonsmooth semi-infinite multiobjective programming, Optim. Lett. 8 (2014), 1517-1528.

[13] N. Kanzi, On strong KKT optimality conditions for multiobjective semi-infinite programming problems with Lipschitzian data, Optim. Lett. 9 (2015), 1121-1129.

[14] S. Kazemi, N. Kanzi, Constraint qualifications and stationary conditions for mathematical programming with non-differentiable vanishing constraints, J. Optim. Theory Appl. 179 (2018), 800-819.

[15] S.K. Mishra, V. Singh, V. Laha, On duality for mathematical programs with vanishing constraints, Annal. Oper. Res. 243 (2016), 249-272.

[16] S.K. Mishra, V. Singh, V. Laha, R.N. Mohapatra, On constraint qualifications for multiobjective optimization problems with vanishing constraints. In: H. Xu, S. Wang, S.Y. Wu (eds.) Optimization Methods, Theory and Applications, pp. 95-135, Springer, Berlin, 2015. 
[17] B. Mond, T. Weir, Generalized concavity and duality. In: S. Schaible, W.T. Ziemba (eds.) Generalized Concavity in Optimization and Economics, pp. 263-279, Academic Press, New York, 1981.

[18] Y. Pandey, S.K. Mishra, On strong KKT type sufficient optimality conditions for nonsmooth multiobjective semi-infinite mathematical programming problems with equilibrium constraints, Oper. Res. Lett. 44 (2016), 148-151.

[19] Y. Pandey, S.K. Mishra, Optimality conditions and duality for semi-infinite mathematical programming problems with equilibrium constraints, using convexificators, Ann. Oper. Res. 269 (2018), 549-564.

[20] R.T. Rockafellar, Convex Analysis, Princeton Math. Ser., vol. 28, Princeton University Press, Princeton, New Jersey, 1970.

[21] L.T. Tung, Karush-Kuhn-Tucker optimality conditions for nonsmooth multiobjective semidefinite and semiinfinite programming, J. Appl. Numer. Optim. 1 (2019), 63-75.

[22] L.T. Tung, Karush-Kuhn-Tucker optimality conditions and duality for semi-infinite programming with multiple interval-valued objective functions, J. Nonlinear Funct. Anal. 2019 (2019), Article ID 22.

[23] L.T. Tung, Karush-Kuhn-Tucker optimality conditions and duality for convex semi-infinite programming with multiple interval-valued objective functions, J. Appl. Math. Comput. 62 (2020), 67-91.

[24] L.T. Tung, Karush-Kuhn-Tucker optimality conditions and duality for multiobjective semi-infinite programming via tangential subdifferentials, Numer. Funct. Anal. Optim. 41 (2020), 659-684.

[25] P. Wolfe, A duality theorem for nonlinear programming, Q. Appl. Math. 19 (1961), 239-244. 